\title{
Functional role of dimerization and CP190 interacting domains of CTCF protein in Drosophila melanogaster
}

Artem Bonchuk ${ }^{1 \dagger}$, Oksana Maksimenko ${ }^{2 \dagger}$, Olga Kyrchanova ${ }^{1 \dagger}$, Tatyana Ivlieva $^{1}, V^{\prime}$ ladic Mogila ${ }^{1,4}$, Girish Deshpande $^{3}$, Daniel Wolle ${ }^{3}$, Paul Schedl ${ }^{2,3^{*}}$ and Pavel Georgiev ${ }^{1 *}$

\begin{abstract}
Background: Insulators play a central role in gene regulation, chromosomal architecture and genome function in higher eukaryotes. To learn more about how insulators carry out their diverse functions, we have begun an analysis of the Drosophila CTCF (dCTCF). CTCF is one of the few insulator proteins known to be conserved from flies to man.

Results: In the studies reported here we have focused on the identification and characterization of two dCTCF protein interaction modules. The first mediates dCTCF multimerization, while the second mediates dCTCF-CP190 interactions. The multimerization domain maps in the N-terminus of the dCTCF protein and likely mediates the formation of tetrameric complexes. The CP190 interaction module encompasses a sequence 200 amino acids long that spans the C-terminal and mediates interactions with the N-terminal BTB domain of the CP190 protein. Transgene rescue experiments showed that a dCTCF protein lacking sequences critical for CP190 interactions was almost as effective as wild type in rescuing the phenotypic effects of a dCTCF null allele. The mutation did, however, affect CP190 recruitment to specific Drosophila insulator elements and had a modest effect on dCTCF chromatin association. A protein lacking the N-terminal dCTCF multimerization domain incompletely rescued the zygotic and maternal effect lethality of the null and did not rescue the defects in Abd-B regulation evident in surviving adult $d C T C F$ mutant flies. Finally, we show that elimination of maternally contributed $d C T C F$ at the onset of embryogenesis has quite different effects on development and $A b d-B$ regulation than is observed when the homozygous mutant animals develop in the presence of maternally derived dCTCF activity.

Conclusions: Our results indicate that dCTCF-CP190 interactions are less critical for the in vivo functions of the dCTCF protein than the N-terminal dCTCF-dCTCF interaction domain. We also show that the phenotypic consequences of dCTCF mutations differ depending upon when and how dCTCF activity is lost.
\end{abstract}

Keywords: Bithorax complex, BTB domain, Chromatin insulator, Embryo development, Transcription

\section{Background}

The chromatin fiber in the chromosomes of multicellular animals is organized into a hierarchical set of topologically independent domains [1-6]. This hierarchical loop domain organization is critical for the proper utilization and propagation of the genetic information encoded by the

\footnotetext{
* Correspondence: pschedl@Princeton.EDU; georgiev_p@mail.ru

${ }^{\dagger}$ Equal contributors

2Laboratory of Gene Expression Regulation in Development, Institute of Gene Biology, Russian Academy of Sciences, Moscow, Russia

'Department of the Control of Genetic Processes, Institute of Gene Biology, Russian Academy of Sciences, Moscow, Russia

Full list of author information is available at the end of the article
}

chromosome and it is intimately involved in such processes as gene regulation, replication, recombination, repair, and mitosis. A special set of architectural elements, called boundaries or insulators, are responsible for both subdividing the chromatin fiber into discrete domains and determining their hierarchical organization. These architectural elements have a seemingly contradictory set of functions. When placed between enhancers/silencers and their target genes, they block regulatory interactions [1, 7-11]. However, insulators also have the ability to promote regulatory interactions between enhancers/silencers and their target genes. One mechanism depends upon

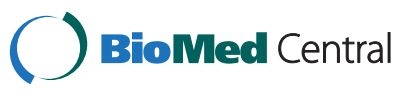

(c) 2015 Bonchuk et al. Open Access This article is distributed under the terms of the Creative Commons Attribution 4.0 International License (http://creativecommons.org/licenses/by/4.0/), which permits unrestricted use, distribution, and reproduction in any medium, provided you give appropriate credit to the original author(s) and the source, provide a link to the Creative Commons license, and indicate if changes were made. The Creative Commons Public Domain Dedication waiver (http://creativecommons.org/publicdomain/zero/1.0/) applies to the data made available in this article, unless otherwise stated. 
insulator pairing interactions that connect distant chromosomal segments together at the base of a topological loop $[12,13]$. When the regulatory elements in the loop are properly oriented, these insulator-insulator pairing interactions can place distant enhancers/silencers in close proximity to target genes. Additionally, there is suggestive evidence that insulators can have an even more direct role in mediating enhancer-promoter interactions [14-21].

Nearly a dozen DNA binding proteins that have insulator/ architectural activity have been discovered. Of these, the most broadly conserved protein is the CCCTC-binding factor, CTCF [22]. Except for a few lineages that have apparently lost the CTCF gene, it is a characteristic feature of bilaterian organisms [23-25]. CTCF was initially identified as a transcription factor that can both repress and activate transcription [26, 27]. Subsequent experiments in flies and vertebrates showed that CTCF also has enhancer-blocking activity and to date it is the only mammalian protein has been shown to have this insulator activity [28-30]. In addition to its roles in gene regulation, a growing body of evidence points to an architectural function. For example, in vertebrates CTCF mediates specific long-distance interactions between insulators and regulatory elements located at megabase distances and even on different chromosomes [31-34]. Similarly, Drosophila dCTCF can support pairing-dependent insulator bypass in transgene assays when two sets of multimerized binding sites for the protein are arranged in the appropriate orientation $[15,35]$.

Like the CTCF proteins of vertebrates, Drosophila CTCF contains $11 \mathrm{C} 2 \mathrm{H} 2$ zinc fingers flanked by $\mathrm{N}$-terminal domains (NTDs) and C-terminal domains (CTDs) [36]. The dCTCF zinc fingers show significant homology with their vertebrate counterparts, and this is reflected in the similar sequence recognition properties of the vertebrate and fly proteins [37]. In contrast, the NTDs and CTDs are not well conserved and there are reasons to think that the differences between the NTDs and CTDs of vertebrate and fly proteins have mechanistic implications. The insulator/architectural activities of vertebrate CTCF depend at least in part upon its ability to recruit the cohesin complex to specific chromosomal sites [38-41]. Cohesin knockdowns were shown to impair both the insulator (enhancer-blocking) and architectural (long-distance interactions) functions of CTCF $[31,42,43]$. The vertebrate cohesin complex consists of four proteins: Smc1, Smc3, Scc1, and SA/STAG [44]. Smc1 and Smc3 form a ring in the presence of ATP, and this ring is stabilized by the binding of Scc1 and SA/STAG. Vertebrate CTCF is thought to recruit cohesins to specific chromosomal sites by interacting directly with the SA/STAG subunit of cohesin complex. Xiao et al. [45] have shown that sequences in the C-terminal tail of CTCF are responsible for specific interactions with SA/STAG, and that insulator activity and cohesin recruitment are disrupted when this region of the CTCF protein is mutated.

dCTCF differs from its vertebrate counterpart in that it does not appear to co-localize with cohesins $[44,46]$. This finding has led to the idea that other proteins might fulfill the long-distance linking function envisioned for cohesins. One plausible candidate is CP190 [47]. It was originally identified as a microtubule binding protein that associates with the centrosome during mitosis [48, 49]. However, subsequent studies argued against a centrosome or mitotic function and instead pointed to a role in some aspect of nuclear architecture or chromosome structure [50, 51]. Support for this idea came from the discovery that CP190 is required for the enhancer-blocking activity of the gypsy $\mathrm{Su}(\mathrm{Hw})$ insulator and is a component both of this transposon insulator and of endogenous $\mathrm{Su}(\mathrm{Hw})$ insulators $[52,53]$. The connection to chromosome architecture was further supported by studies showing that CP190 localizes to many dCTCF sites and can be co-immunoprecipitated with dCTCF [54-57].

In the studies reported here we have examined the functioning dCTCF protein in more detail. Using a combination of biochemical and genetic approaches we have identified the CP190 interaction domain. We also uncovered a dCTCF dimerization/multimerization domain that, like CP190, could potentially mediate interactions between distant DNA sequences containing dCTCF in vivo. In the course of this analysis we have reexamined the effects of dCTCF null mutations, and tested whether dCTCF proteins lacking the CP190 interaction domain or the $\mathrm{ACTCF}$ dimerization/multimerization domain can rescue the null mutation.

\section{Results}

\section{dCTCF contains an $\mathrm{N}$-terminal dimerization domain}

The 11 zinc fingers of the CTCF proteins are highly conserved in bilaterian phyla [24, 25] (see schematic in Fig. 1a and Additional file 1: Figure S1A). In contrast, the NTDs and CTDs were poorly conserved and there was little sequence similarity even between proteins from different dipteran families (Additional file 1: Figure S1A). Sequence alignment of CTCF proteins from species within the Drosophila genus revealed much more extensive homology in the NTDs and CTDs, including several very well conserved sequence blocks (Additional file 1: Figure S1B). A plausible hypothesis is that these conserved sequences may serve as protein interaction modules that are important for dCTCF activities.

One of the interactions that could be mediated by these modules was the dimerization or multimerization of the dCTCF protein. This possibility was suggested by insulator bypass experiments in which pairs of multimerized and appropriately oriented CTCF binding sites could mediate long-distance regulatory interactions $[15,16]$. 


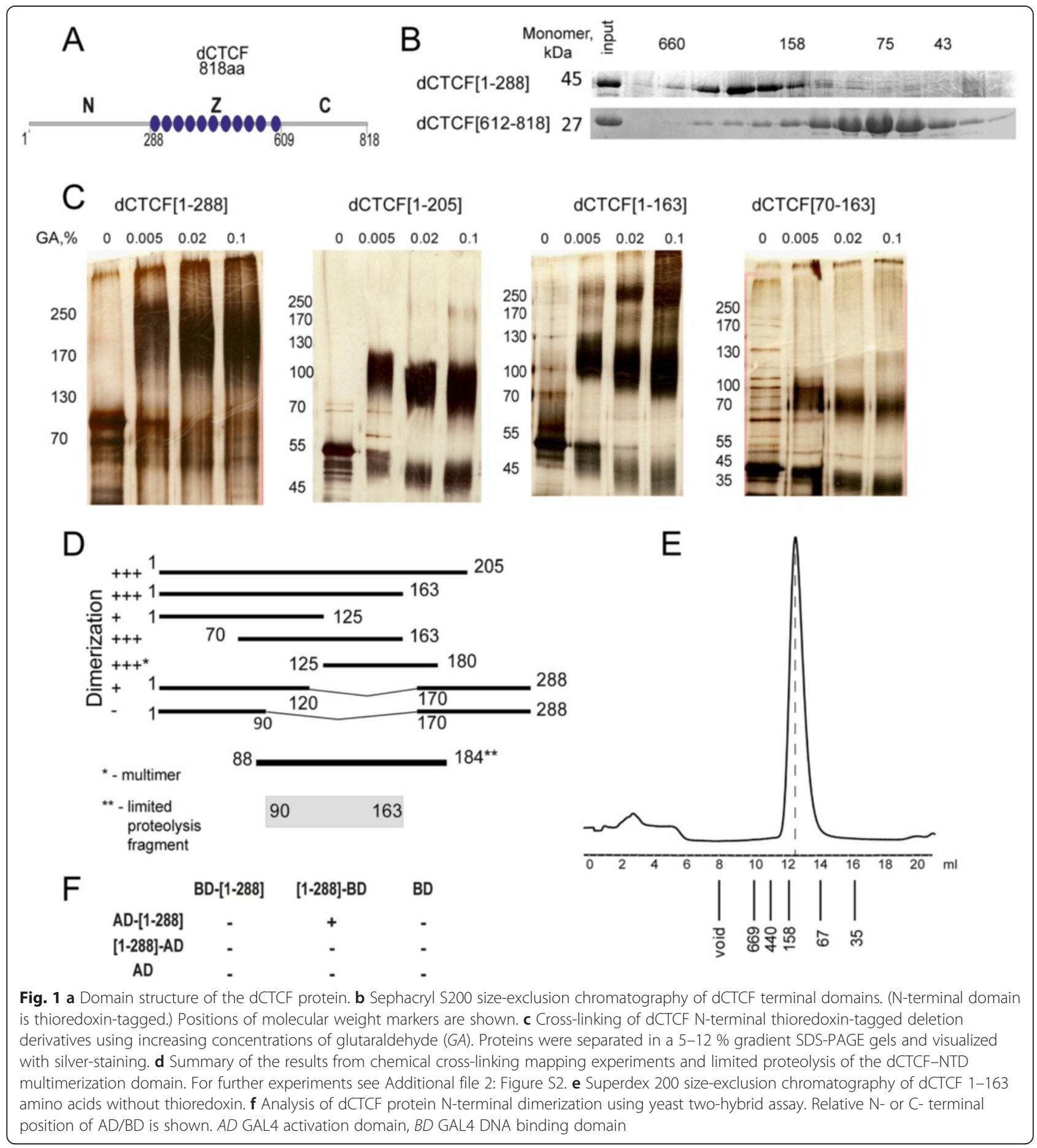

Additionally, studies on vertebrate CTCF have suggested that it can form dimers $[58,59]$. To test for the presence of homo-dimerization/multimerization modules in the $\mathrm{N}$ - or $\mathrm{C}$-terminus of the $\mathrm{dCTCF}$ protein, we fractionated bacterially expressed thioredoxin-fused NTD or CTD proteins by size-exclusion chromatography. As shown in Fig. 1b, the thioredoxin NTD fusion 1-288 had a hydrodynamic molecular mass significantly larger $(\sim 250$
$\mathrm{kD}$ ) than that predicted for the monomer (45 kD). Similar results were obtained for the CTD 612-818 protein (Fig. 1b).

The presence of these larger complexes could be explained by either a module-dependent multimer formation or by the presence of intrinsically disordered regions that lead to non-specific protein aggregation. To distinguish between these possibilities we used glutaraldehyde cross- 
linking to probe for complex formation. In the case of the CTD 612-818 protein, glutaraldehyde cross-linking was quite inefficient, suggesting that it likely forms nonspecific aggregates (Additional file 2: Figure S2A). On the other hand, consistent with the results of the sizeexclusion chromatography, cross-linking of the NTD 1-288 protein gave a high yield of a multimeric band of $\sim 200 \mathrm{kD}$ (Fig. 1c).

To further pinpoint the interaction module, we generated three C-terminal deletions (see Fig. 1d). The smallest deletion, NTD 1-205, gave a cross-linked band of $\sim 100 \mathrm{kD}$. The largest C-terminal deletion, NTD 1-125, also gave a cross-linked product; however, the yield was quite low compared to the NTD 1-205 protein (compare Additional file 2: Figure S2A to Fig. 1c). This suggests that key interaction sequences were located between amino acids 125 and 205. This suggestion was supported by NTD 1-163, which was much more efficiently cross-linked than NTD 1-125 (Fig. 1c). Though NTD 1-163 gave a prominent cross-linked band at the approximate size expected for the tetramer $(\sim 120 \mathrm{kD})$, there was also a ladder of larger bands. This ladder was likely due, at least in part, to the presence of the thioredoxin moiety in the fusion protein, as only two cross-linked bands were observed when thioredoxin was removed (Additional file 2: Figure S2A). Taken together, these findings map the $\mathrm{N}$-terminal dCTCF:dCTCF multimerization module to sequences spanning the region between amino acids 125 and 163 and suggest that this module likely mediates the formation of dimers or possibly tetrameric complexes. Further support for the formation of multimeric complexes (tetrameric or an even larger) came from size-exclusion chromatography of the NTD 1-163 protein (lacking the thioredoxin moiety), which gave a predicted mass of $120 \mathrm{kD}$ (Fig. 1e). However, it is also possible that disordered regions of the protein retard complex mobility during size-exclusion chromatography.

Several additional lines of evidence localized the dCTCF multimerization module to this region of the NTD. First, two internal deletions (Fig. 1d) that lacked sequences from this interval failed to cross-link efficiently (Additional file 2: Figure S2A). Second, two terminally truncated proteins, NTD $125-180$ and NTD 70-163 (Fig. 1d), that contained this part of the NTD were cross-linked efficiently (Additional file 2: Figure S2A). Third, protease digestion indicated that the region containing the interaction module had an ordered structure. We subjected the thioredoxin NTD 1-205 fusion protein to limited proteinase $\mathrm{K}$ or trypsin digestion and then analyzed the resulting protease-resistant products by matrix-assisted laser desorption/ionization time-offlight (MALDI-TOF) mass spectrometry (Additional file 2: Figure S2B; Additional file 3: Table S1). Both proteases generated two resistant-to-digestion products. One corresponded to thioredoxin, while the other to a dCTCF NTD peptide extending from 84 to 188 .

To independently demonstrate that the NTD contains a dCTCF multimerization module we used two different in vivo assays. The first was a yeast two-hybrid assay (Fig. 1f). Sequences encoding the NTD 1-288 amino acids were fused in-frame to the yeast GAL4 DNA binding domain (BD) and activation domain (AD). Because steric hindrance can interfere with transcriptional activation in the two-hybrid system, the NTD 1-288 sequence was placed at both the N-terminus (NTD-AD and NTD-BD) and the C-terminus (AD-NTD and BD-NTD) of the fusion protein. Fig. 1f shows that activation was observed in only one configuration, NTD-BD and AD-NTD. Similar results were obtained when the NTD was tested with a full-length dCTCF protein (not shown).

In the second assay, we ectopically expressed a 3xFLAGtagged fusion protein consisting of the N-terminal 302 amino acids of dCTCF, a nuclear localization signal and the bacterial LexA DNA BD in Drosophila S2 tissue culture cells. The S2 cells were co-transfected with a plasmid encoding the firefly luciferase protein, whose expression is dependent upon a minimal TATA-box promoter and upstream 4xLexA binding sites (Fig. 2a). Measurements of luciferase activity relative to a Renilla luciferase co-transfection control indicated that the 3xFLAG-N-terminal dCTCF-LexA fusion protein weakly activated firefly luciferase expression from the 4xLexA-TATA reporter (Fig. 2b). By contrast, no activation was observed for a luciferase reporter that lacked the 4xLexA binding sites or when the 3xFLAG-tagged fusion protein had the LexA DNA BD but not the dCTCF N-terminal domain. The chromatin immunoprecipitation (ChIP) experiments in Fig. 2c show that when the N-terminal dCTCF fusion protein was tethered to the 4xLexA-TATA reporter via the LexA $B D$, it could interact with and recruit endogenous full-length dCTCF. Because CP190 antibodies were also able to immunoprecipitate the 4xLexA-TATA reporter, it would appear that the full-length dCTCF protein could in turn recruit CP190 to the 4xLexA-TATA reporter (via the CTD of the full-length dCTCF protein: see below).

\section{The $\mathrm{BTB}^{\mathrm{CP} 190}$ dimer interacts with the $\mathrm{C}$-terminal domain of $\mathrm{dCTCF}$}

CP190 has an N-terminal BTB-POZ (BTB for BR-C, ttk and bab and POZ for Pox virus and Zinc finger) protein-protein interaction domain, which is followed by an aspartic acid-rich D domain, a microtubule targeting domain, four $\mathrm{C} 2 \mathrm{H} 2$ zinc fingers (which bind nonspecifically to DNA), and finally a glutamic acid rich $\mathrm{C}$ terminal domain (Fig. 3a). Previously it was shown that the CP190 protein interacts with dCTCF $[54,55]$. While interacting modules in the two proteins were not identified, it was found that the BTB domain is required for the binding of CP190 to chromatin [60]. 
A

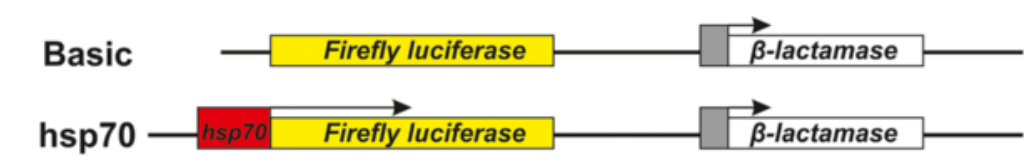

TATA

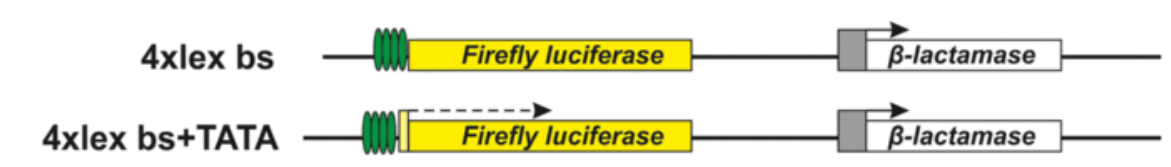

B

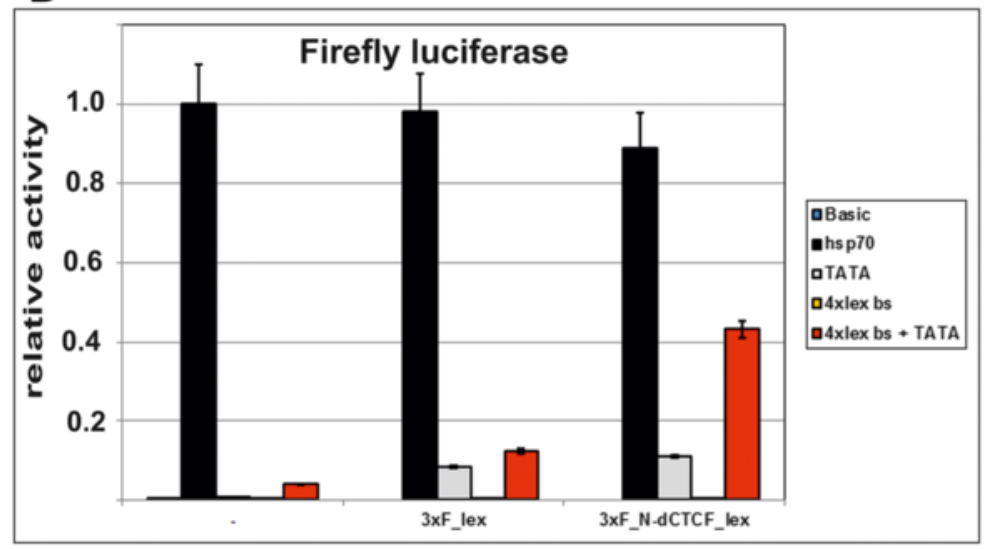

C

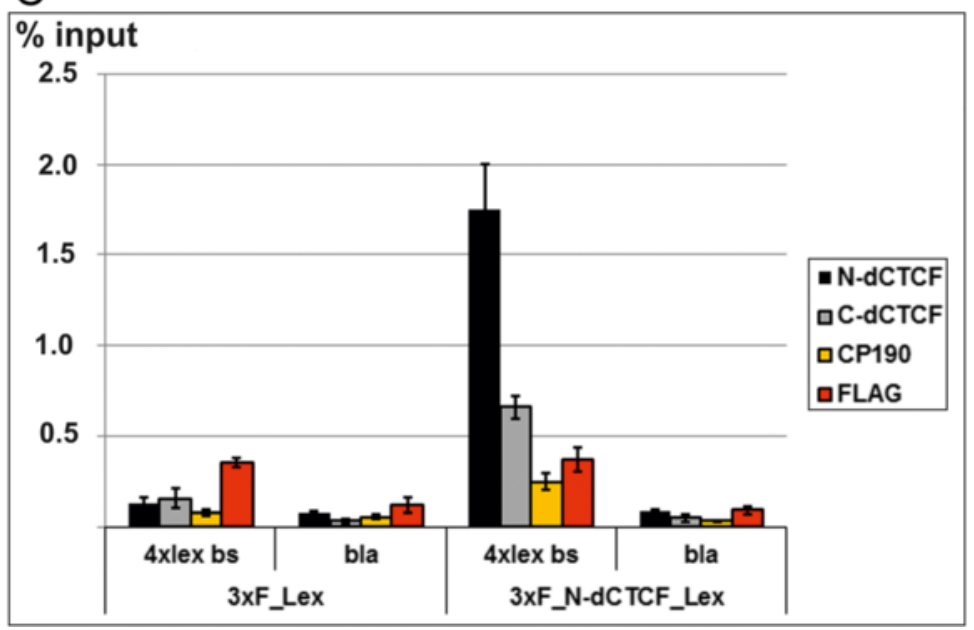

Fig. 2 a Schematic drawing of luciferase reporter constructs. $\mathbf{b}$ Firefly luciferase expression from the five reporters shown in a when co-transfected with empty vector, with a vector encoding a 3xFLAG-tagged-(nuclear localization signal)-LexA fusion protein, or with a vector encoding the 3xFLAG-tagged $\mathrm{N}$-terminal dCTCF-(nuclear localization signal)-LexA fusion protein. A plasmid encoding the Renilla luciferase under the control of the actin promoter was used to correct for variations in transfection efficiency, and expression of the firefly luciferase was normalized in each case to Renilla luciferase. Each transfection experiment was performed in three independent biological replicates and each lysate was measured in four technical replicates. Error bars show standard deviations of measurements of all summarized replicates. c Chromatin immunoprecipitation of S2 cells co-transfected with the 4xLesA TATA-box reporter or the basic promoterless reporter and either of two fusion protein expression constructs, the 3xFLAG-tagged (nuclear localization signal) LexA construct or the 3xFLAG-tagged-N-terminal dCTCF-(nuclear localization signal)-LexA construct. Fixed and processed S2 chromatin samples were immunoprecipitated with antibodies directed against (as indicated) the dCTCF N-terminus, the dCTCF C-terminus, CP190, or FLAG, and then assayed for the presence of sequences corresponding to the 4xLexA TATA reporter or the basic reporter constructs as indicated. Each chromatin immunoprecipitation experiment was performed in three independent biological replicates. Error bars show standard deviations of summarized biological replicates after quadruplicate PCR measurements in each experiment. The results are presented as a percentage of input DNA. Basic no promoter, bla basic promoterless reporter, Hsp70 firefly luciferase with an hsp70 promoter, TATA firefly luciferase with a minimal TATA-box promoter, $4 x$ lex bs, firefly luciferase with four copies of the LexA recognition sequence, 4xlex bs + TATA, firefly luciferase with four copies of the LexA recognition sequence linked to a minimal TATA-box promoter 


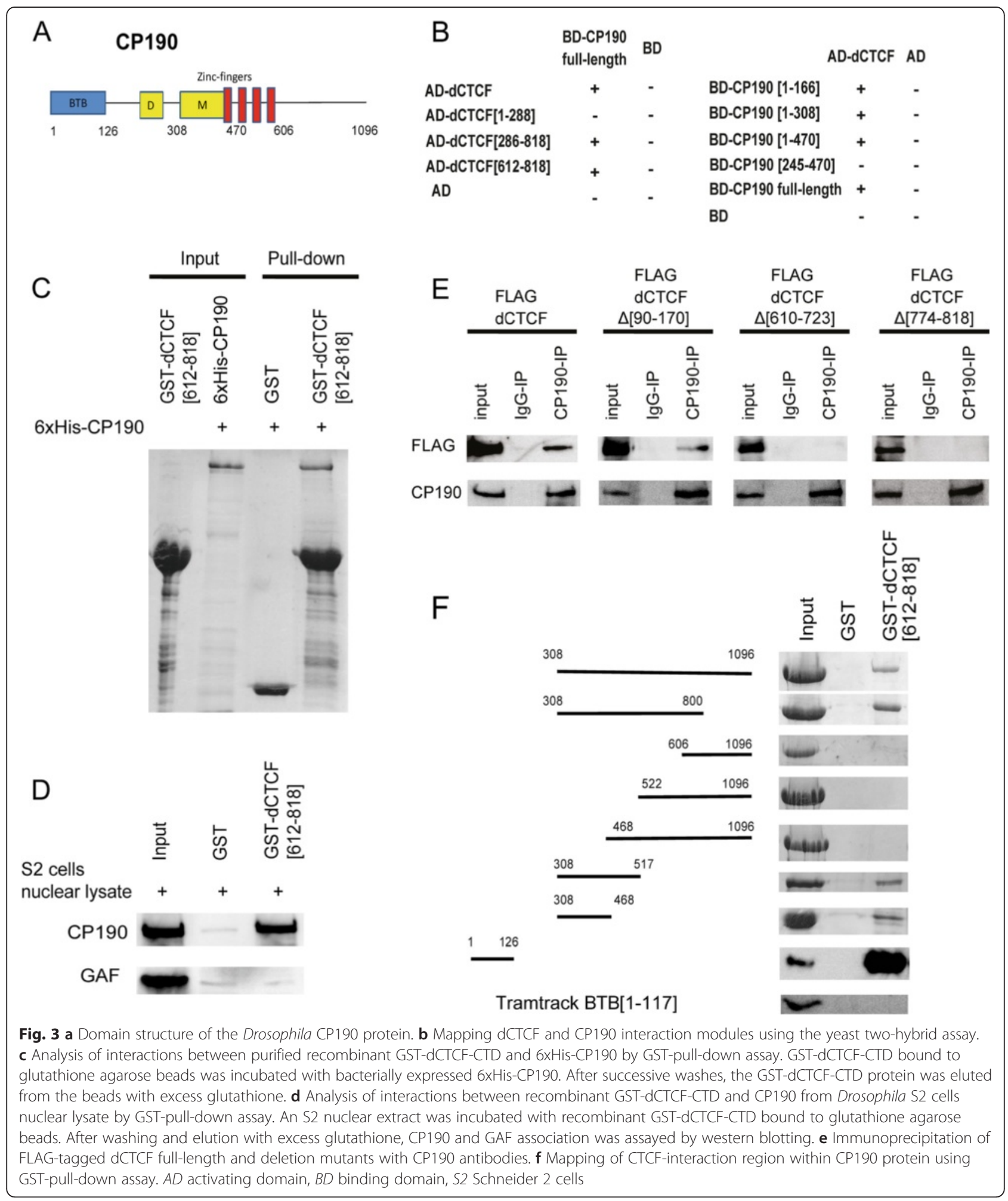

To identify modules in dCTCF that mediate CP190 interaction, we first used the yeast two-hybrid assay. dCTCF was subdivided into the NTD, the zinc fingers plus the CTD, and the CTD alone and each was fused to the GAL4 activation domain. The NTD failed to interact, while the full-length protein, the zinc fingers plus the CTD, and the CTD alone gave transcriptional activation when combined with full-length CP190 (Fig. 3b). The localization of the CP190 interaction module in dCTCF to the CTD was confirmed by GST pull-down experiments. 
A GST-fusion protein containing the dCTCF-CTD 612818 domain was found to pull down both bacterially expressed CP190 and CP190 in Drosophila S2 cell nuclear extracts (Fig. 3c,d).

To further pinpoint the sequences within the 612-818 CTD that are important for contacting CP190, we expressed two different FLAG-tagged C-terminal deletions, dCTCF $\Delta 610-723$ and dCTCF $\Delta 774-818$, in Drosophila S2 cells. Figure 3e shows that FLAG-tagged wild-type dCTCF and a control N-terminal deletion could be precipitated by CP190 antibodies from the S2 extracts. In contrast, neither of the smaller dCTCF-CTD deletions was precipitated by CP190 antibodies from S2 cells. Taken together, these findings indicate that an apparently rather large sequence is required to mediate a dCTCF-CP190 association that is stable in $\mathrm{S} 2$ nuclear extracts.

We used a similar strategy to localize the region in the CP190 protein that mediates interactions with dCTCF. For the yeast two-hybrid experiments, full-length dCTCF was fused to the GAL4 activation domain, while different sub-fragments from CP190 were fused to the GAL4 DNA $\mathrm{BD}$. These experiments map a dCTCF interaction module to the CP190 BTB domain (Fig. 3b). This was confirmed by GST pull-down experiments (Fig. 3f) which showed strong protein-protein interactions between the CP190 BTB domain (1-126) and dCTCF. In addition, weak interactions were detected between the dCTCF-CTD and GST-CP190 fusions spanning the microtubule interaction domain (see CP190 308-517 and 308-468 in Fig. 3f).
We have previously shown that the CP190 BTB domain exists as a stable homodimer [61]. This observation raised the possibility that a CP190 dimer could simultaneously bind two dCTCF proteins, linking them together in the same manner that the Bcl6 BTB dimer is thought to bring together two SMRT co-repressors [62]. However, glutaraldehyde cross-linking experiments argue that the predominant complex consists of a $\mathrm{BTB}^{\mathrm{CP} 190}$ dimer linked to a single CTD protein. Figure 4a shows that the CP190 BTB domain alone formed a stable dimer that could be readily captured by glutaraldehyde cross-linking. When the BTB domain was present in a twofold excess over the dCTCF-CTD 612-818 protein, the cross-linked $\mathrm{BTB}^{\mathrm{CP} 190}$ dimer disappeared and was replaced by a band migrating with an apparent molecular weight of $\sim 130 \mathrm{kD}$. While this cross-linked complex migrated more slowly than we would have predicted, we interpret it to be a $2 \mathrm{xBTB}^{\mathrm{CP} 190}$ :CTD $612-818$ trimer based on the stoichiometry of the two proteins. This conclusion was supported by cross-linking experiments in the presence of increasing amounts of the CTD protein (Fig. 4b). At a CTD to BTB ratio of $1: 4$ and 1:2, the predominant cross-linked species was the $\sim 130 \mathrm{kD} 2 \mathrm{xBTB}^{\mathrm{CP} 190}$ :CTD trimer, whereas there was little if any of the $\mathrm{BTB}^{\mathrm{CP} 190}$ dimer. Only at ratios of 1:1 or $2: 1$ did we observe a larger species that could correspond to the $\mathrm{BTB}^{\mathrm{CP} 190}$ dimer linked to two CTD proteins or to some other more complex structure(s). However, under these conditions a significant fraction of the CTD protein appeared to be free monomer, and this

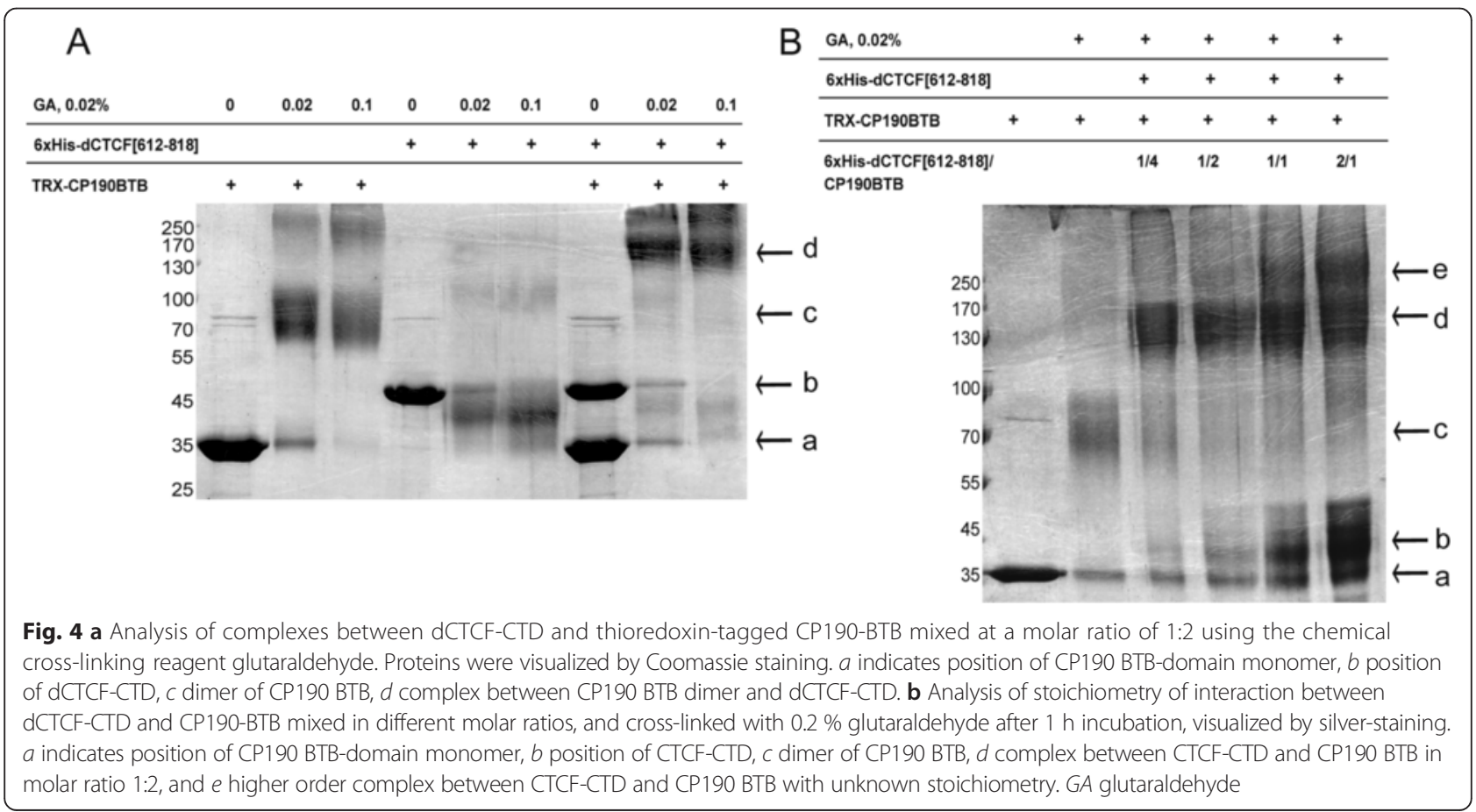


would also argue that the preferred configuration is a $2 \mathrm{xBTB}^{\mathrm{CP} 190}$ :CTD heterotrimer.

\section{Rescuing a dCTCF null allele}

If the NTD and CTD interaction modules are critical for dCTCF function, then dCTCF proteins that lack these modules should be defective. As a prelude to assaying the activity of NTD and CTD mutant proteins in vivo, we determined whether $d C T C F$ mutant flies could be rescued by a transgene expressing the wild-type $\mathrm{dCTCF}$ protein. Several putative $d C T C F$ null alleles have been reported $[54,55,63]$. Flies homozygous for these mutations $\left(d C T C F^{30.6}, d C T C F^{Y+1}, d C T C F^{Y+2}, d C T C F^{30}\right)$ mainly died during larvae-pupae stages. For the wildtype rescue construct we generated P-element transformants of a hybrid fusion gene that expresses the $d C T C F$ cDNA under the control of the ubiquitously expressed hsp83 promoter [64, 65]. To identify the transgene proteins, a sequence encoding a 3xFLAG epitope was introduced at the beginning of the $\mathrm{dCTCF}$ open reading frame. Five independent transgene inserts were recovered on the first and second chromosomes; however, none of these transgenes rescued the lethal effects of the four $d C T C F$ alleles. One reason why the transgenes were unable to complement the four $d C T C F$ alleles is that they did not express as much protein as the endogenous $d C T C F$ gene. An alternative possibility is that there were additional lethal lesions on the chromosomes carrying these particular $d C T C F$ mutations.

A fifth predicted $d C T C F$ null allele, GE24185, has been described [55]. The viability of adults homozygous for the GE24185 mutation is reduced by a third or more, while F2 flies do not survive. The GE24185 mutation was generated by insertion of an $\mathrm{EP}^{\mathrm{S}}$ transposon in reverse orientation into the third exon of the $d C T C F$ gene (Fig. 5a). The $\mathrm{EP}^{\mathrm{S}}$ transposon contains an $h s p 70$ minimal promoter that drives transcription in the opposite orientation to the $d C T C F$ gene [66]. The promoter is under control of a GAL4-responsive enhancer. As would be expected from its insertion site, the GE24185 disrupts expression of the dCTCF protein. Extracts prepared from F1 adults homozygous for the GE24185 mutation showed no dCTCF-specific bands when probed with antibodies directed against $\mathrm{N}$-terminal or $\mathrm{C}$-terminal regions of the dCTCF protein (Fig. 5b). Unlike the other $d C T C F$ alleles, we found that two copies of the hsp83$d C T C F^{+}$transgene rescued the F1 and F2 lethal phenotypes of the GE24185 mutation.

To confirm these findings, we generated two imprecise excisions, GEx52 and GEx56, by introducing the P transposase. As indicated in Additional file 4: Figure S3, both imprecise excisions disrupted the coding sequence and were expected to encode only a truncated protein containing the first $\sim 158 \mathrm{~N}$-terminal amino acids. Both excision derivatives had the same phenotypic effects as GE24185 and were complemented by the hsp83-dCTCF transgene. These results support the conclusion that GE24185 is a null allele of the $d C T C F$ gene [55].

As was shown previously [55], adult flies homozygous for the GE24185 mutation as well as the two excision derivatives had a mild but highly penetrant held out wing phenotype and thin bristles throughout the animal, and exhibited a series of homeotic phenotypes in posterior parasegments indicative of a loss of $A b d-B$ activity. These homeotic phenotypes were temperature dependent. They were typically observed in flies raised at $25{ }^{\circ} \mathrm{C}$, while they were much less frequent when the flies were raised at $18{ }^{\circ} \mathrm{C}$. One of these phenotypes was the presence of a rudimentary A7 segment in males as would be expected for a loss-of-function transformation of PS12 into PS11 (Fig. 5d). Another was a protruding and rotated male genitalia. Also unlike wild-type males, GE24185 males had bristles on the A6 sternite and sometimes also patchy pigmentation of the A5 tergite. The former phenotype is characteristic of a PS11 to PS10 transformation, while the latter is expected for a PS10 to PS9 transformation. While A7 and A8 do not form cuticular structures in adult males, they contribute to the cuticle in females. Homeotic transformations of the A7 sternite into A6 were evident in surviving GE24185 females (Additional file 5: Figure S4). Adult mutant females had significantly reduced egg production, and produced no viable offspring when mated to homozygous mutant males. These lethal effects could, however, be rescued by mating the homozygous mutant females to heterozygous balancer males. This finding indicates that zygotic $d C T C F$ expression can compensate for the absence of maternally derived dCTCF.

\section{Selective depletion of dCTCF from the bithorax complex in GE241845 pupae}

Mohan et al. (2007) found that though the levels of dCTCF were substantially reduced in GE24185 larvae, maternally derived protein could still be detected at approximately $25 \%$ of the sites in salivary gland polytene chromosomes that are normally observed in wild-type polytenes [55]. One idea suggested by this observation is that the homeotic transformations evident in GE24185 adults arise because dCTCF is selectively lost from the bithorax complex (BX-C). When dCTCF depletion compromises BX-C insulator function, this might enable Polycomb response elements (PREs) in silenced cis-regulatory domains to repress neighboring active cis-regulatory domains and thus downregulate $A b d-B$ expression in a manner that changes segmental identity. Alternatively, or in fact in addition, the proper functioning of the $A b d-B$ promoter could require dCTCF.

Mohan et al. [55] addressed this question by examining $\mathrm{dCTCF}$ association with BX-C in the brain of wild- 


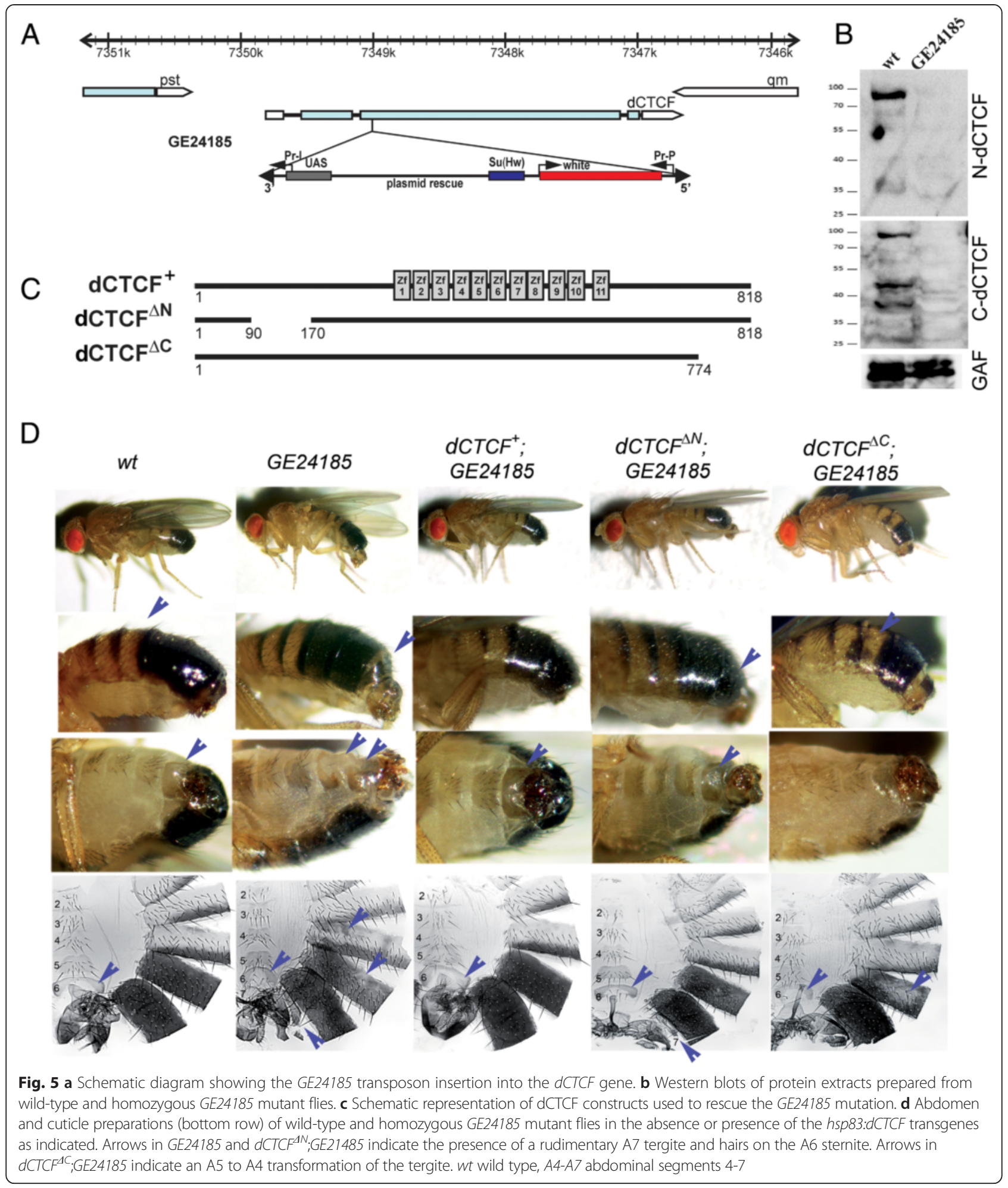

type and GE24185/Df(3L)0463 larvae and found that dCTCF was absent from most insulators in the complex, but was detected at $A b d-B$ promoter. We have repeated these experiments using chromatin prepared from pupae because it is during this stage that the adult cuticle is elaborated. We selected six dCTCF binding sites from BX-C: the Mcp, Fab-6, Fab-8 insulators [67-77], Fab-3 region, Fab-4 region, and the $A b d-B$ promoter region (Fig. 6) [78]. We also selected the CG1354 promoter region (9A1) [55] and four regions that were identified by 


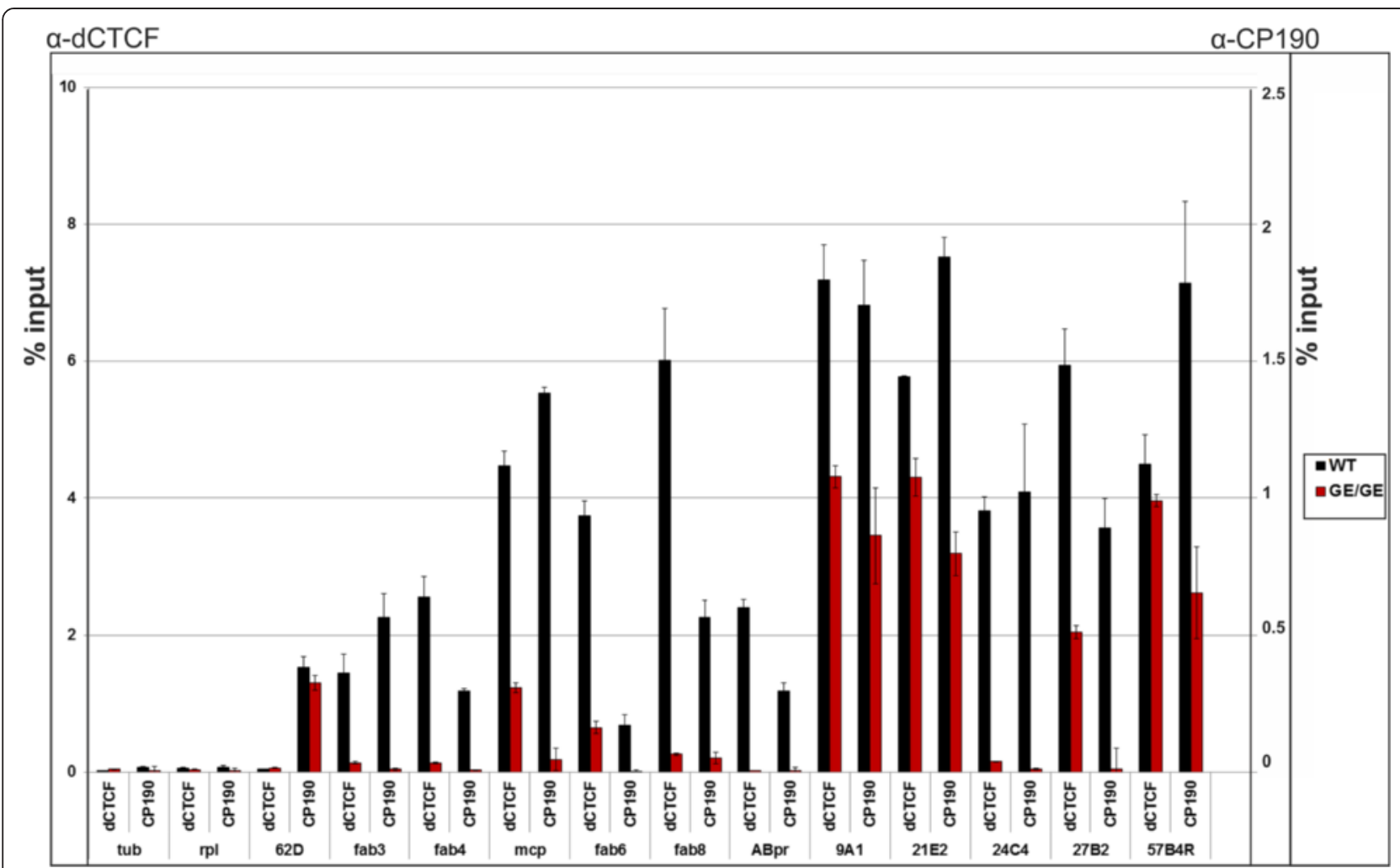

Fig. 6 Histograms show dCTCF or CP190 occupancy in chromatin isolated from mid-late pupa at sequences containing the BX-C insulators Fab-3, Fab-4, Mcp, Fab-6, Fab-8, the Abd-D promoter, and several previously defined dCTCF insulators (9A1, 21E2, 24C4, 27B2 and 57B4R). Cross-linked chromatin prepared from wild-type (WT) $\left(y^{1} w^{1118}\right)$ pupae and homozygous GE24185 (GE/GE) mutant pupae was immunoprecipitated with antibodies directed against the N-terminal domain of dCTCF and CP190. Sequences from tub, rpl32, and 62D regions were used as negative controls for dCTCF and CP190 association. 62D is an example of a sequence in which CP190 occupancy is independent of dCTCF. The left axis shows the scale for dCTCF enrichment, while the right axis shows the scale for CP190 enrichment. Each chromatin immunoprecipitation experiment was performed in at least two independent biological replicates. Error bars show standard deviations of quadruplicate PCR measurements. The results are presented as a percentage of input DNA

Schwartz et al. [56] as requiring dCTCF to block the spread of H3K27me3 in the BGL3 cell line [79]. In addition to testing $\mathrm{dCTCF}$ association with these sequences, we also assayed CP190.

ChIP experiments with chromatin isolated from wildtype pupae using antibodies directed against the $\mathrm{N}$ terminal region of dCTCF confirmed that it was bound to the insulators and the $A b d-B$ promoter in $\mathrm{BX}-\mathrm{C}$ and to the CG1354 promoter (9A1) and four BGL3 insulators (Fig. 6). However, the extent of enrichment of Fab-3, Fab-4, and the $A b d-B$ promoter was about half that of the other BX-C insulators ( $M c p, F a b-6$, and Fab-8) and also of the CG1354 promoter (9A1) and four BGL3 insulators. As expected, the enrichment of the BX-C dCTCF sequences was substantially reduced in ChIPs of homozygous GE24185 pupae. The extent of reduction was not, however, uniform. Near-background levels of dCTCF were observed for $F a b-3, F a b-4, F a b-8$, and the $A b d-B$ promoter in GE24185 mutant pupae, while residual dCTCF could still be detected at Mcp and, to a lesser extent, Fab-6. By contrast, only one BGL3 insulator, 24C4, showed a loss of
dCTCF equivalent to that seen for most of the BX-C sequences. Three of the other insulators, 9A1, 21E2, and 57B4R, showed only very modest reductions. Though the loss of dCTCF at the fourth insulator, 27B2, was more substantial, the occupancy level in mutant pupae was still about the same as that seen for several of the BX-C elements in wild type.

The rather modest reductions in $\mathrm{dCTCF}$ evident at several non-BX-C insulators as well as the residual dCTCF that was retained at two of the BX-C insulators in the absence of a zygotic source of dCTCF protein would suggest that a significant amount of maternally derived dCTCF remains up to at least the pupal stage in GE24185 mutant animals. Moreover, it would appear that the protein is preferentially retained at a subset of the $d C T C F$ insulators. However, an alternative explanation for the apparent persistence of maternal dCTCF is that our antibody recognized some other protein species that happened to bind to the insulators that were pulled down in ChIPs of the mutant pupae. To exclude this possibility, we used an antibody directed against the C- 
terminal region of the dCTCF protein for ChIP experiments (Additional file 6: Figure S5). ChIPs with this antibody paralleled those obtained with the $\mathrm{N}$-terminal antibody. Substantial amounts of dCTCF persisted at several non-BX-C insulators in GE24185 mutant pupae, while there was still some residual dCTCF remaining at the BX-C insulators Mcp and Fab-6.

With the exception of $M c p$, all of the BX-C insulators and the $A b d-B$ promoter had less CP190 than the BGL3 insulators. The effects of GE24185 mutation on CP190 association with the BX-C and BGL3 sequences also followed a pattern similar to that observed for dCTCF. For all of the BX-C insulators, loss of dCTCF was accompanied by a loss of CP190. For the other insulators, the reduction in CP190 occupancy was, with one exception, roughly comparable to that seen at the insulator for dCTCF. For example, dCTCF levels were reduced about $40 \%$ for 9A1, while CP190 was reduced about $50 \%$. The one exception was 27B2, which lacked CP190 in GE24185 mutant pupae, yet retained significant dCTCF occupancy. To confirm that the loss of CP190 occupancy at dCTCF insulators was not due to a reduction in CP190 protein levels, we probed western blots of extracts prepared from wild-type and GE24185 mutant pupae (Additional file 7: Figure S6).

\section{Role of CTDs and NTDs in functional activity of the dCTCF protein}

To examine the in vivo functions of the N-terminal multimerization domain and the C-terminal CP190 interacting domain, we generated $h s p 83$ transgenic lines expressing FLAGx3-tagged dCTCF proteins lacking these domains. For the multimerization domain, we deleted sequences between amino acid 90 and amino acid $170\left(\mathrm{dCTCF}^{\Delta \mathrm{N}}\right)$. This deletion spans the region required for $\mathrm{dCTCF}-\mathrm{dCTCF}$ interactions in vitro. For the CP190 interaction module, we used the C-terminal 774-818 deletion $\left(\mathrm{dCTCF}^{\Delta \mathrm{C}}\right)$ that eliminates interactions between CTCF and CP190 in S2 cells (Fig. 5c). The activities of two independent transgenic lines expressing the deleted proteins were tested in the GE24185 mutant background.

As described above, the control transgene, $d C T C F^{+}$, encoding the wild-type protein fully complemented the zygotic and maternal effect lethality of the GE24185 mutation (Fig. 5d). It also rescued the thin bristles phenotype and the loss-of-function homeotic transformations evident in PS11-14 (Fig. 5 and Additional file 5: Figure S4). However, in approximately $10 \%$ of the $\mathrm{dCTCF}^{+}$males we observed a partial loss of pigmentation in the tergite of abdominal segment A5, which is characteristic of a loss-of-function transformation of A5 (PS10) to A4 (PS9) transformation [80]. The held out wing phenotype was also not rescued. The $d C T C F^{\Delta C}$ transgenes resembled $d C T C F^{+}$. They fully rescued the zygotic and maternal effect lethality of the
GE24185 mutations, the thin bristles, and the PS11-14 homeotic transformations in males and females. Like $d C T C F^{+}$, we also observed a partial loss of pigmentation on the A5 tergite; however, the frequency was somewhat higher ( $50 \%$ as compared to $10 \%$ ) and the size of the depigmented patches was typically larger. In contrast to $d C T C F^{\Delta C}$, the $d C T C F^{\Delta N}$ transgene only partially ameliorated the zygotic and maternal effect lethality of GE24185 and $d C T C F^{\triangle N}$ transgenic flies had reduced viability and were only semi-fertile. In addition, $d C T C F^{\Delta N}$ did not rescue the thin bristles phenotype or the homeotic transformation seen in the abdominal segments of GE23185 adult males and females (Fig. 5d and Additional file 5: Figure S4).

\section{Chromatin association of $\mathrm{dCTCF}^{+}, \mathrm{dCTCF}^{\Delta \mathrm{C}}$, and $\mathrm{dCTCF}^{\mathrm{\Delta N}}$}

As a prelude to analyzing the chromosome association of the mutant dCTCF proteins, we first examined the expression of transgenic wild-type and mutant dCTCF proteins. For this purpose we probed fly extracts with antibodies directed against the FLAG tag. As shown in the western blot in Additional file 8: Figure S7, the mutant proteins were expressed at nearly equivalent levels. When we probed western blots with antibodies directed against $\mathrm{dCTCF}$, we found that the levels of proteins produced by the transgenes were about twofold less than that of the endogenous gene (not shown). This would suggest that the incomplete rescue of two of the GE24185 phenotypes (loss of A5 pigmentation and held out wings) by the $h s p 83: d C T C F^{+}$transgene is likely due, at least in part, to the insufficient expression of dCTCF.

Next, we examined the association of transgenic wildtype and deletion mutant $\mathrm{dCTCF}$ with the insulators and $A b d-B$ promoter in BX-C, CG1354 promoter (9A1), and the BGL3 insulators. In ChIPs of chromatin isolated from GE24185 hsp83:dCTCF $F^{+}$pupae we found that the occupancy levels of the transgenic $\mathrm{dCTCF}^{+}$at most of these sites were reduced about twofold compared to the endogenous protein in wild-type flies (Fig. 7). This reduction would be consistent with the lower levels of dCTCF in the GE24185 hsp83:dCTCF ${ }^{+}$flies. However, there were three exceptions. For two of these, the Fab-6 and Fab-8 insulators, the reductions in dCTCF occupancy were greater than twofold. dCTCF occupancy at Fab-6 was reduced by nearly tenfold while it was reduced by almost fourfold at Fab-8. While Fab-8 still retained levels of dCTCF comparable to several other BX-C sites, only a small amount of dCTCF was detected at $F a b-6$. Because one function of the Fab-6 insulator in PS10 cells is to prevent inactivation of the $i a b-5$ cis-regulatory domain by blocking the spread of Polycombdependent silencing from the PRE in the adjacent iab-6 cis-regulatory domain, the substantial reduction in dCTCF association with the Fab-6 insulator could potentially 


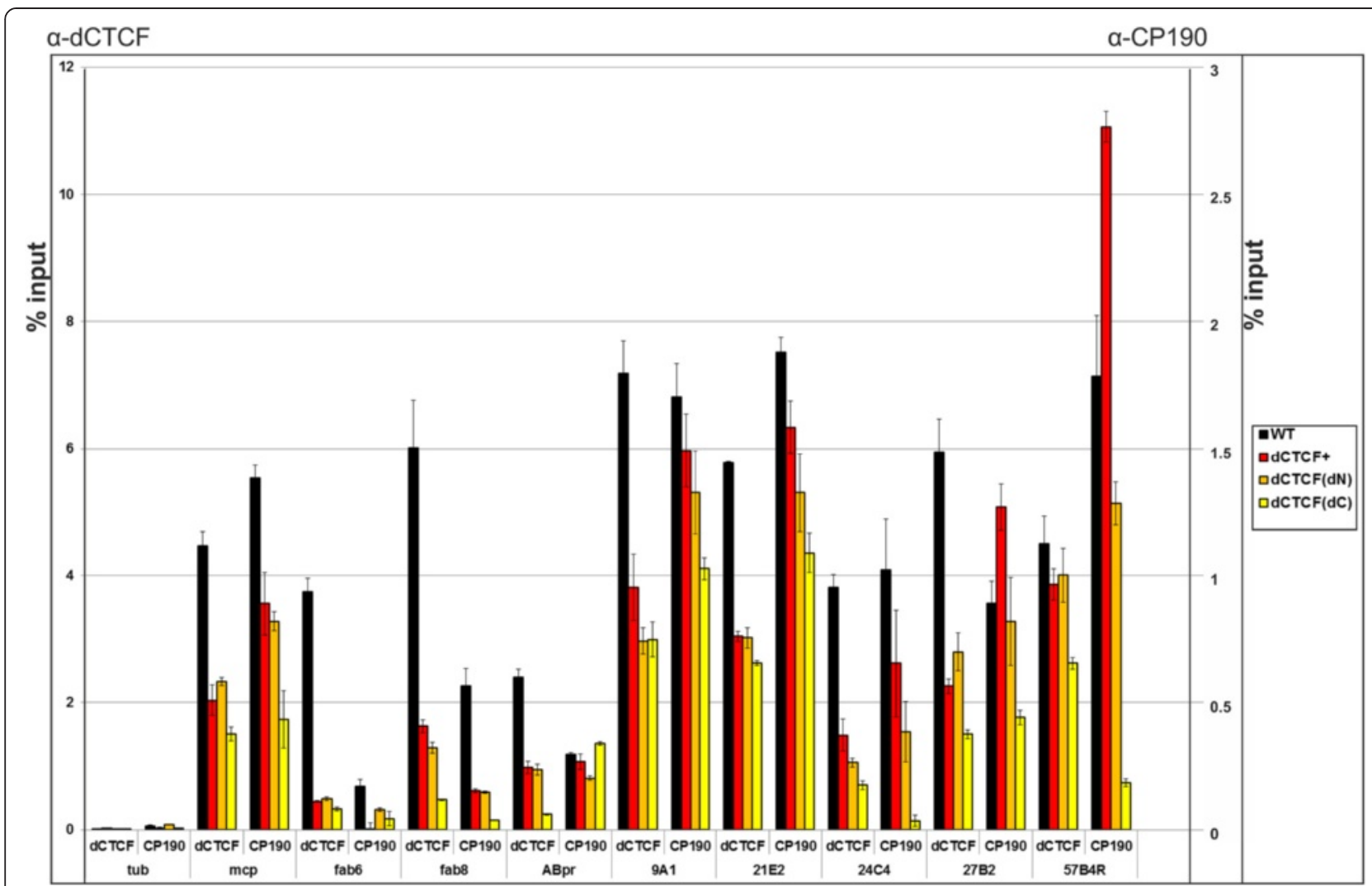

Fig. 7 Histograms show dCTCF or CP190 occupancy in chromatin from mid-late pupa at sequences containing the BX-C insulators Mcp, Fab-6, Fab-8, the Abd-B promoter, and several other previously defined dCTCF insulators (9A1, 21E2, 24C4, 27B2, 57B4R). Chromatin was isolated from homozygous GE24185 mutant pupae that also carry the hsp83:dCTCF ${ }^{+}$, hsp83:dCTCF ${ }^{\Delta N}$, or $h s p 83: d C T C F^{4 C}$ transgenes. The tub sequence was used as the negative control. The left axis shows the scale for dCTCF enrichment, while the right axis shows the scale for CP190 enrichment. Each ChIP experiment with 2- to 3-day pupae was performed in at least two independent biological replicas. Error bars show standard deviations of quadruplicate PCR measurements. The results are presented as a percentage of input DNA. WT wild type

account for the persistence of the A5-A4 transformation in a subset of the GE24185 flies rescued by $h s p 83: d C T C F^{+}$. The other exception, the BGL 57B4R insulator, had near wild-type levels of dCTCF.

Like $d C T C F^{+}, d C T C F^{\Delta N}$ and $d C T C F^{\Delta C}$ occupancy at $\mathrm{dCTCF}$ sites in BX-C and the BGL3 insulators was reduced compared to wild type (Fig. 7). At most sites, the levels of $d C T C F^{\Delta N}$ occupancy were very close to those for $d C T C F^{+}$. In contrast, $d C T C F^{\Delta C}$ occupancy levels were in most instances slightly lower (less than twofold) than either $d C T C F^{+}$or $d C T C F^{\Delta N}$. Though the effects were small, they suggest that chromatin association of the dCTCF C-terminal deletion was partially compromised. Because this domain mediates interactions with CP190, this finding would support the idea that dCTCF binding to chromatin can be stabilized by interactions with CP190.

\section{CP190 occupancy requires dCTCF but not necessarily the dCTCF-CTD}

We also tested whether the reductions in CP190 occupancy evident in GE24185 mutants could be rescued by the $h s p 83: d C T C F$ transgenes encoding the wild-type and mutant proteins. Supporting the idea that dCTCF functions in CP190 recruitment, we found that $d C T C F^{+}$and $d C T C F^{\Delta N}$ promote CP190 occupancy at sites bound by dCTCF in vivo (Fig. 7). For BX-C insulators the effects on CP190 occupancy seemed to correlate with the levels of the transgene dCTCF associated with the insulator. For example, at $M c p$ where the $d C T C F^{+}$and $d C T C F^{\Delta N}$ transgene proteins were present at only about half the level of the endogenous dCTCF, CP190 occupancy was about $60 \%$ of wild type (see Fig. 7). Similarly at Fab-8, the transgene $\mathrm{dCTCF}$ proteins and $\mathrm{CP} 190$ were present at levels about $30 \%$ that of wild type. CP190 occupancy for two BGL3 insulators, 24C4 and 27B2, also depended upon dCTCF. In GE24185 mutants, CP190 was not detected at either of these insulators, while association was restored by the $d C T C F^{+}$and, to a somewhat lesser extent, the $d C T C F^{\Delta N}$ transgenes. Because the three other insulators (9A1, 21E2, and 57B1R) retained significant levels of both dCTCF and CP190 in GE24185 mutants, it was not clear whether dCTCF is essential for CP190 
occupancy or if it is one of several factors that contribute to CP190-insulator association. Thus, though CP190 occupancy in $d C T C F^{+}$and $d C T C F^{\Delta N}$ flies at these three insulators was near wild type, the extent to which transgene dCTCF protein contributed to the rescue was not entirely clear.

Further insight into the role of dCTCF in CP190 occupancy came from ChIPs of $d C T C F^{\Delta C}$ transgene embryos (Fig. 7). There seemed to be three classes with respect to the requirement for the dCTCF-CTD. In the first class were the BGL3 insulators $24 \mathrm{C} 4$ and $54 \mathrm{~B} 4 \mathrm{R}$ and also Fab-8. In this class, CP190 occupancy required the dCTCF-CTD and was substantially reduced in $d C T C F^{\triangle C}$ transgene flies compared to wild type or the two other $d C T C F$ rescue transgenes (Fig. 7). The second class was represented by $M c p$ and 27B2. Like 24C4, 54B4R and Fab-8, CP190 occupancy at these two insulators depended upon dCTCF and was reduced to nearbackground levels in GE24185 flies. However, unlike the insulators in the first class, the $d C T C F^{\Delta C}$ transgene could partially rescue CP190 association. In the third class was the $A b d-B$ promoter. Although CP190 occupancy at the $A b d-B$ promoter required dCTCF (see Fig. 6), the requirement seemed to be independent of the dCTCF-CTD and was fully rescued by the $d C T C F^{\triangle C}$ transgene.

\section{dCTCF is required to properly initiate Abd-B expression in the embryo}

The visible phenotypic defects in GE24185 adult flies arise from alterations in the patterns of gene expression induced by the gradual depletion of maternal dCTCF as the animals develop. It seemed possible that the effects on gene regulation might differ if dCTCF were completely absent at the onset of embryonic development instead of being present at near-normal levels and then slowly lost. To explore this possibility, we examined the expression of three genes, the homeotic gene $A b d-B$, the segment polarity gene, engrailed (en) [81], and the Notch pathway gene, insensitive (insv) [82] in the progeny of GE24185 mothers and fathers. Unlike the progeny of heterozygous parents, these embryos lack both maternal and zygotic dCTCF. Because the greatly reduced fecundity of GE24185 mothers made embryo collections problematic, we restricted our analysis to mid-embryogenesis.

The pattern of Abd-B expression during midembryogenesis in wild-type embryos is dynamic [83, 84]. In stage 10 germ band extended embryos, Abd-B protein is expressed in parasegments PS13 and PS14, while little or no protein is evident in more anterior parasegments. Abd-B protein first begins to accumulate at detectable levels in more anterior parasegments towards the end of stage 11 at the onset of germ band retraction. Only a low level of protein is initially observed in PS12. As the germ band retracts, Abd-B levels increase in PS12, and protein begins to accumulate at detectable levels in PS11. Finally at the end of germ band retraction in stage 13, low levels of Abd-B are found in PS10. Panels E-G in Fig. 8 show the pattern of Abd-B expression in a stage $10 d C T C F^{m-z^{-}}$embryo. For the purposes of comparison, a slightly older stage 11 wild-type embryo is shown in panels A-C. Abd-B expression in the $d C T C F^{m-z-}$ embryo differed in two respects from wild type. First, the levels of Abd-B in both PS13 and PS14 of the $d C T C F^{m-z_{-}}$embryo were noticeably higher than that found in the corresponding parasegments of the wild-type embryo (compare panels C and G). Second, while Abd-B could not be detected in PS12 in the stage 11 wild-type embryo, it was prematurely expressed in PS12 in the stage $10 d C T C F^{m-z-}$ mutant embryo. The differences in both timing and level of expression seen in stage 10/11 wild-type and $d C T C F^{m-z-}$ embryos were also evident in older embryos. In the stage 12 wild-type embryo shown in Fig. 9a,b, there was at most only a very low level of Abd-B protein in PS12, while Abd-B did not appear to be expressed in PS11. In contrast, Abd-B was readily detected in both PS12 and PS11 of the $d C T C F^{m-z-}$ embryo. Moreover, protein could even be seen in a cluster of cells in PS10. In addition to being prematurely expressed in more anterior parasegments, the level of Abd-B protein in PS13 and PS14 was higher than that in the wild-type control [83, 84].

The segment polarity gene en and the Notch pathway gene insv were expressed in a stripe-like pattern in each parasegment in the ectoderm of wild-type germ band extended embryos; however, while all of the cells in the en stripes appeared to express essentially the same levels of En protein (Fig. 9d,h) only a subset of the cells in the insv stripes expressed Insv (Figs. 8d and 9c). In the case of En, there were no obvious changes in the stripe pattern or in the level of protein in the cells expressing En in the $d C T C F^{m-z-}$ mutant. In contrast, there was a substantial increase in the number of cells that expressed Insv in $d C T C F^{m-z-}$ embryos. The level of Insv protein in these cells also appeared to be elevated. These changes were evident in both the stage 10 embryo in Fig. $8 \mathrm{~h}$ and the stage 12 embryo in Fig. $9 \mathrm{~h}$.

We also examined the expression of Abd-B in GE24185 embryos rescued by the $h s p 83: d C T C F^{+}, h s p 83: d C T C F^{\Delta N}$, and $h s p 83: d C T C F^{\triangle C}$ transgenes. The pattern of Abd-B expression in GE24185 hsp83: dCTCF $F^{+}$transgenic embryos resembled wild type. This was also true for GE24185 dCTCF ${ }^{\triangle C}$ transgenic embryos. In the case of hsp83:dCTCF ${ }^{\Delta N}$, we occasionally observed stage 11-15 embryos that appeared to have slightly elevated levels of Abd-B.

\section{Discussion}

CTCF is one of only a few insulator/chromosomal architectural DNA binding proteins that are known to be 


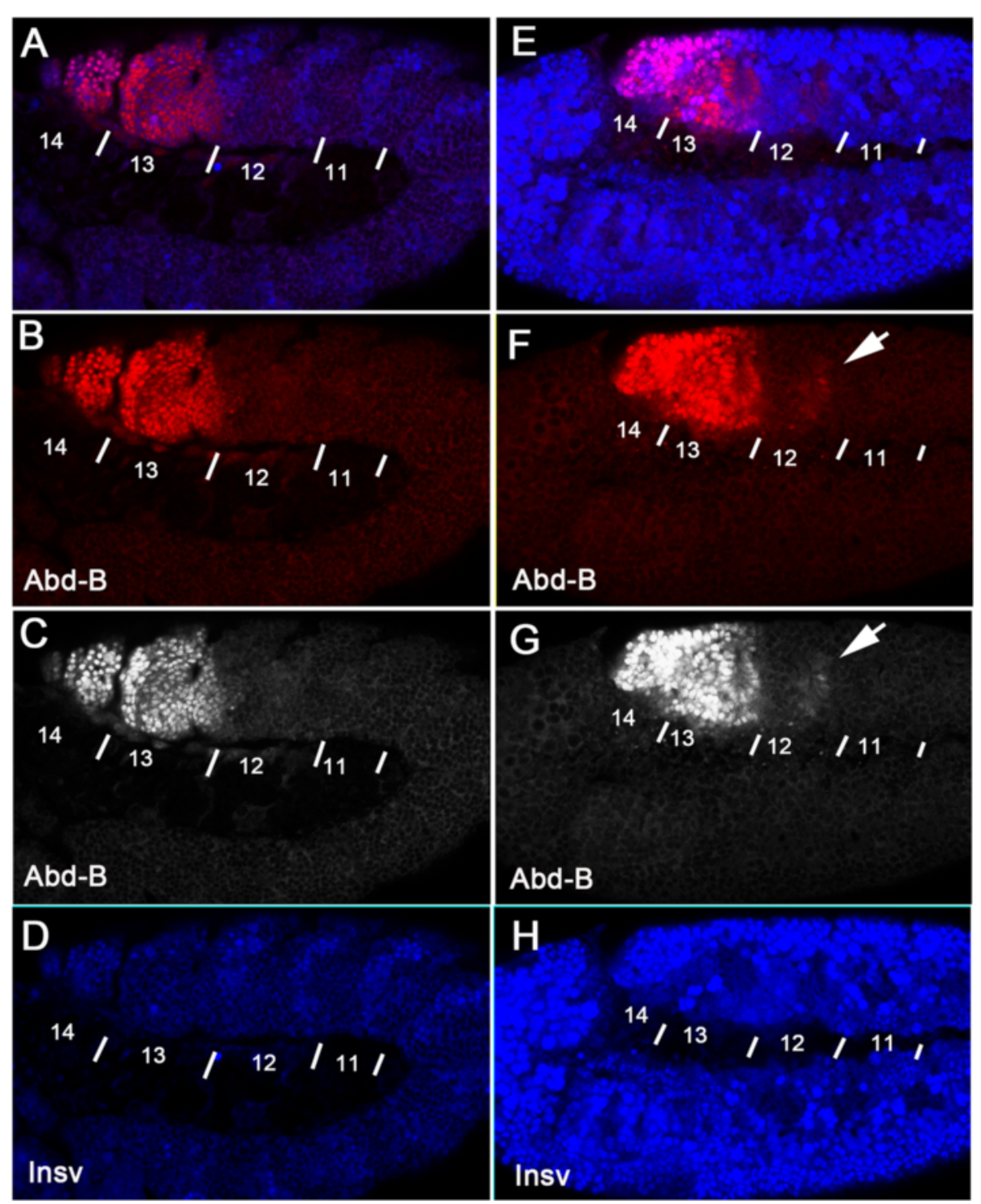

Fig. 8 Expression of Abd-B and Insv in stage 10/11 wild-type and dCTCF $F^{m-z^{-}}$embryos. Stage 11 wild-type and stage 10 dCTCF ${ }^{m-z^{-}}$(from cross of homozygous GE24185 parents) embryos were probed with antibodies directed against Abd-B (mouse monoclonal 1A2E9 from Developmental Studies Hybridoma Bank) and Insv (a rabbit polyclonal: gift of Tsutomu Aoki) and visualized by confocal microscopy. Parasegments are indicated in the Fig. Arrows in panels F and G indicate Abd-B expression in PS12. a Wild type: merged image. b Wild type: Abd-B. c Wild type: Abd-B. d WT: Insv. e $d C T C F^{m-z^{-}}$: merged image. f $d C T C F^{m-z^{-}}:$Abd-B. g dCTCF ${ }^{m-z^{-}}:$Abd-B. h dCTCF $F^{m-z^{-}}$: Insv. Red/Gray Abd-B, Blue Insv

common to both insects and vertebrates [28, 30, 36, 85]. While it has been implicated in a wide range of nuclear functions ranging from gene expression to recombination and replication, a thread that seems common to most of its known activities is one of organizing the chromatin fiber. With the aim of better understanding its biological activities, we have begun a systematic analysis of the Drosophila melanogaster CTCF protein, $\mathrm{dCTCF}$. In the studies reported here we have focused on two modules in the protein, one that mediates multimerization of $\mathrm{dCTCF}$ and the other that mediates interactions with the chromosomal protein CP190.
Using a combination of biochemical procedures we localized the dCTCF multimerization module to the $\mathrm{N}$ terminus of the protein, spanning a region of about 100 amino acids between 70 and 163. At least part of this region (84-188) appears to be highly structured in solution as it is protease resistant. Although further studies will be required, our data would be most consistent with a model in which the multimerization module mediates the formation of tetrameric rather than dimeric dCTCF complexes. However, in either case, multimerization should enable dCTCF to interact simultaneously with several closely spaced recognition motifs because is 


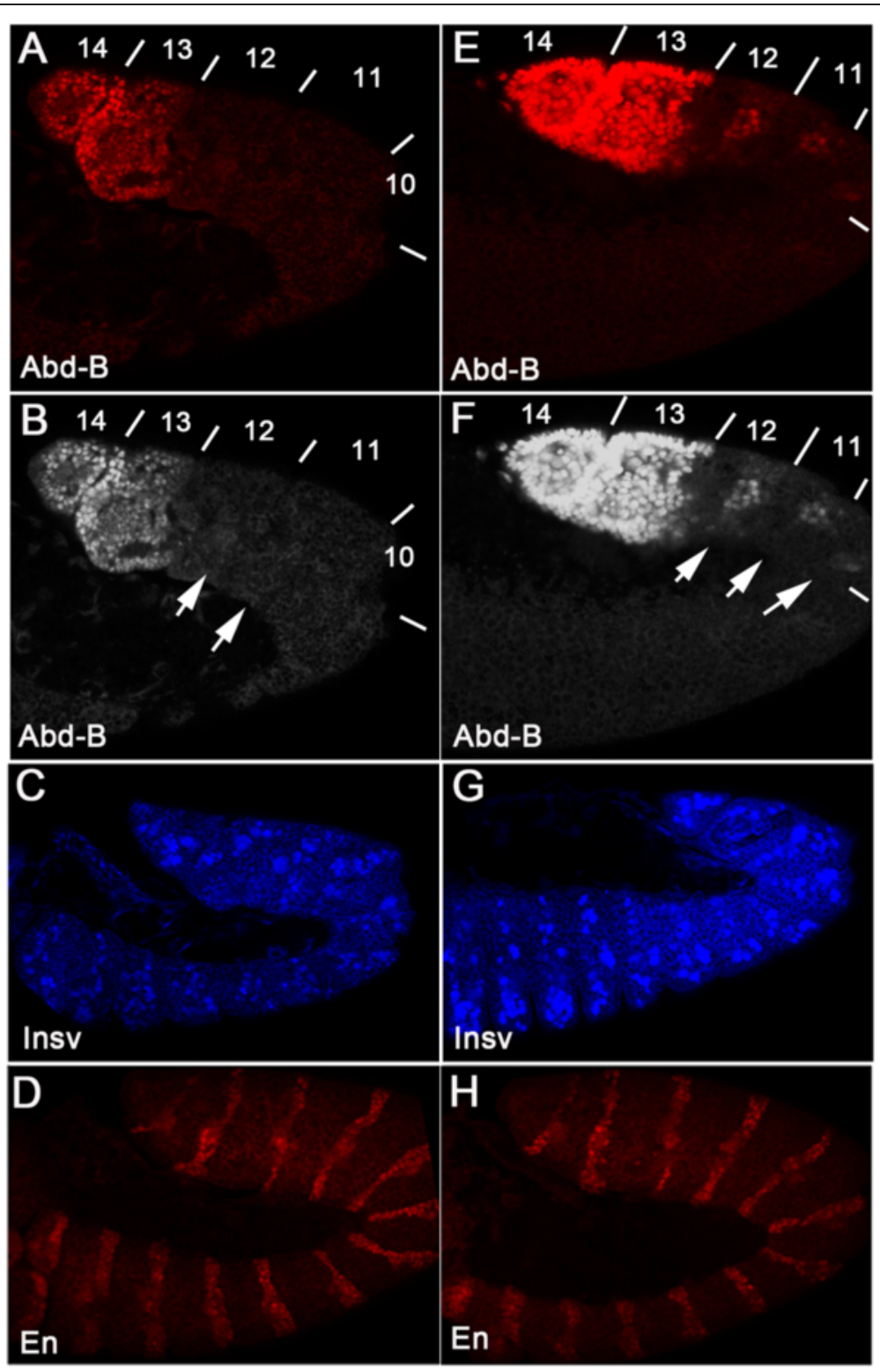

Fig. 9 Expression of Abd-B, Insv, and En in stage 12 wild-type and $d C T C F^{m-z^{-}}$embryos. Stage 12 wild type (a-d) and dCTCF ${ }^{m-z^{-}}$(e-h) were probed with antibodies directed against Abd-B (panels a,b e, and f), Insv (panels $\mathrm{c}$ and g), and En (panels $\mathrm{d}$ and $\mathrm{h}$ ). Arrows in panel $\mathrm{f}$ point to Abd-B protein expression in PS12, PS11, and PS10 in the stage 12 dCTCF ${ }^{m-z^{-}}$embryo. By contrast, arrows in panel b indicate that little or no Abd-B was detected in PS12 or PS11 of the wild-type embryo. See text for details

found in insulators like Fab-3, Fab-6, and Fab-8. Presumably this could stabilize dCTCF association with chromatin by increasing the half-life of the proteinDNA complex. Alternatively, or in addition, the dCTCF multimers could bind simultaneously to dCTCF sites in different insulators, generating a chromatin loop linked together at its base by the insulator bound dCTCF multimeric complexes. A direct linkage of distant dCTCF binding sites by the dCTCF multimers would be consistent with the insulator bypass experiments using pairs of the appropriately oriented multimerized dCTCF binding sites [15]. Like its insect counterpart, the vertebrate CTCF protein has also been implicated in the formation of chromatin loops [28]. Because vertebrate CTCF is capable of self-association [58], it is possible that distant CTCF binding sites are linked together via some sort of CTCF multimeric complex. However, unlike the NTD of the Drosophila protein, biochemical studies suggest that the NTD of vertebrate CTCF is monomeric and disordered [86]. Thus, multimer formation would have to involve other domains. In this regard it is of interest that Pant et al. [59] found pairwise 
interactions between the $\mathrm{C}$-terminal zinc finger domains of the vertebrate CTCF protein.

We also analyzed how dCTCF and CP190 interact with each other. Yeast two-hybrid experiments and GST-pull-downs map the CP190 interaction module of dCTCF to the CTD. For CP190, the primary dCTCF interaction module was associated with the CP190 BTB domain. Previous studies have shown that the CP190 BTB domain forms a highly stable dimer [51, 61]. If there is a one-to-one relationship between the dCTCFCTD-CP190 interaction module and the CP190 BTB domain, this CP190 BTB dimer could link two dCTCF multimers together, potentially building higher order protein-DNA complexes. However, our experiments do not favor a one-to-one interaction between $\mathrm{ACTCF}$ and CP190. Instead, our results would support a model in which a single dCTCF-CTD-CP190 interaction module associates preferentially with a dimerized CP190 BTB domain to give a $2 \times \mathrm{xTB}^{\mathrm{CP} 190}$ : $\mathrm{dCTCF}-\mathrm{CTD}$ heterotrimer. This appears to be true, at least in these in vitro experiments, even when dCTCF is present in excess. Though confirmation will require a direct structural analysis of the dCTCF-CP190 complex, these findings would argue that the BTB dimer of CP190, by itself, could not function as the bridge linking two distant $\mathrm{dCTCF}$ insulators together. However, CP190 has additional domains (aspartic acid-rich $\mathrm{D}$ and microtubule targeting domains) that are involved in interaction with the DNA binding proteins like the insulator protein ZIPIC [60, 87]. Thus, CP190 might form complexes with or bridges between different insulator proteins like ZIPIC and dCTCF that might stabilize binding to chromatin. Because CP190 has a sequence non-specific zinc finger DNA BD [52], an alternative function for CP190 might be to stabilize dCTCF association with chromatin by binding (non-specifically) to sequences adjacent to dCTCF binding sites. However, because there are only very modest effects at most on dCTCF occupancy when the C-terminal dCTCF CP190 interaction domain is deleted, such a function would not seem to be critical.

To complement these biochemical studies, we examined the functioning of the dCTCF multimerization and CP190 interaction modules in vivo. Previous studies have shown that maternally derived $\mathrm{dCTCF}$ is sufficient to sustain development through to the adult stage [55]. However, the viability of homozygous GE24185 animals, which lack zygotic $d C T C F$, was significantly reduced and the surviving adult flies exhibited several characteristic phenotypes. These included thin bristles, held out wings, abdominal segmentation defects, a substantially reduced egg production, and a maternal effect lethality. We found that an $h s p 83$ transgene expressing the wild-type protein rescued all but two of the phenotypic effects of the GE24185 mutation. The two exceptions were the held out wing phenotype and the presence, in a small fraction of the $h s p 83: d C T C F^{W T}$ adults, of a weak A5 (PS10) to A4 (PS9) transformation. It seems likely these two remaining phenotypes were due to the fact that the level of dCTCF expressed by the transgene is less than that produced by the endogenous gene. In the case of the A5 to A4 transformation, this suggestion fits with both the roughly tenfold reduction in dCTCF occupancy at the Fab-6 insulator and the known role of this insulator in $A b d-B$ regulation. Fab-6 functions to protect the $A b d-B$ cis-regulatory domain specifying PS10 (A5), $i a b-5$, from the adjacent PS11 cis-regulatory domain, $i a b-6[69,77,88,89]$. When only reduced levels of dCTCF are present, it is possible that Polycomb group (PcG) complexes may spread into $i a b-5$ from $i a b-6$ silencing the $i a b$-5 cis-regulatory domain inappropriately in PS10.

Somewhat surprisingly, a dCTCF protein, $\mathrm{dCTCF}^{\Delta \mathrm{C}}$, lacking sequences in the CTD critical for interactions with CP190 was just about as effective as the wild-type protein in complementing the GE24185 mutant. Like wild type, it rescued the zygotic and maternal effect lethality, the egg production defect, and the bristle phenotypes, but not the held out wing or the A5 to A4 transformation. ChIP experiments were consistent with the idea that CP190 interactions may help stabilize dCTCF association with chromatin because dCTCF occupancy levels were reduced in $h s p 83: d C T C F^{\Delta C}$ pupae (compared to $h s p 83: d C T C F^{W T}$ ) at most of the insulators we examined. While the effects of the mutation on dCTCF occupancy were modest, much greater reductions in CP190 levels were evident at least at some sites (e.g., the BGL3 insulators, $24 \mathrm{C}$ and 57B4R; Fig. 6). At these sites it would appear that CP190 occupancy required the dCTCF C-terminal CP190 interaction module. However, this was not always the case. For example, at $M c p$, the presence of the mutant $\mathrm{dCTCF}^{\Delta \mathrm{C}}$ protein was sufficient to ensure CP190 occupancy (Fig. 6). Because CP190 occupancy at Mcp required dCTCF (see Fig. 5), it would appear that dCTCF can promote CP190 association by a mechanism that is independent of its Cterminal CP190 interaction module. Recently, Pita was found to bind Mcp and recruit CP190 [87]. The binding of the two proteins to $M c p$ are interdependent. One obvious possibility is that $\mathrm{dCTCF}^{\Delta \mathrm{C}}$ binding to $M c p$ stabilizes the association of Pita, which in turn can recruit CP190.

While the $\mathrm{dCTCF}^{\Delta \mathrm{C}}$ protein was just about as effective as wild-type dCTCF in rescuing the GE24185, this was not true for $\mathrm{dCTCF}^{\triangle \mathrm{N}}$. This mutant protein only partially ameliorated the zygotic and maternal effect lethality, and surviving adult flies exhibited the same visible phenotypes as the GE24185 mutant. Because occupancy levels of the $\mathrm{dCTCF}^{\Delta \mathrm{N}}$ protein were nearly equivalent to that of $\mathrm{dCTCF}^{\mathrm{WT}}$, it would appear that some other activity of the dCTCF protein must be partially compromised 
by the deletion of the multimerization domain. One possibility would be a function in linking distant $\mathrm{dCTCF}$ insulators together; however, this region of the protein could have other activities besides mediating dCTCF dimerization. Importantly, because CP190 occupancy at BX-C insulators and the $A b d-B$ promoter was similar to that in hsp83:dCTCF ${ }^{W T}, A b d-B$-dependent abdominal phenotypes in adult hsp83:dCTCF ${ }^{\Delta N}$ flies (and, by inference, GE24185 flies) may arise by a mechanism that is independent of CP190.

Because there is a substantial maternal contribution of dCTCF, the abdominal segmentation defects in surviving GE24185 flies were likely a consequence of a gradual reduction in dCTCF occupancy at the $A b d-B$ insulators and/or promoter as the animals developed. Significantly, eliminating $\mathrm{dCTCF}$ at the onset of embryonic development had a quite different effect on $A b d-B$ gene activity. Instead of being reduced, $A b d-B$ expression was substantially upregulated in the posterior parasegments of $d C T C F^{m-z-}$ embryos. These paradoxical effects on $A b d-B$ regulation would not be consistent with a primary function for dCTCF in the intrinsic activity of the $A b d-B$ promoter-the adult phenotype would require dCTCF to function as an activator, while the embryonic phenotype would require $\mathrm{dCTCF}$ to function as a repressor. Rather, one would imagine that whatever role dCTCF plays at the $A b d-B$ promoter (positive or negative), this function is likely to be the same throughout development. For this reason, the opposing stage-specific effects on $A b d-B$ activity are more readily explained by the quite different modes of regulation of the $A b d-B$ ( $i a b)$ cis-regulatory domains in embryos and in larvae/pupae [88, 89].

In larvae and pupae, $A b d-B$ regulation is in the maintenance phase and depends upon the PREs in each $i a b$ cis-regulatory domain [73, 88-91]. The PREs function to recruit PcG proteins, keeping inactive cis-regulatory domains off in the parasegments where they should be silenced. For this reason, a gradual reduction in insulator activity in the larval and pupal stages could result in the spreading of PcG silencing from PREs in inactive cisregulatory domains to their flanking active neighbors and the consequent downregulation of $A b d-B$ gene activity in posterior parasegments. (Of course, if $d C T C F$ were also required for $A b d-B$ promoter activity, this would tend to enhance any loss-of-function phenotypes associated with the spread of silencing in the cis-regulatory domains in $d C T C F^{z-}$ mutants. Conversely, these loss-of-function phenotypes would be suppressed if $d C T C F$ represses instead of enhances $A b d-B$ promoter activity). By contrast, in $d C T C F^{m-z-}$ embryos, dCTCF would be absent when the gap and pair-rule genes initially establish the parasegmentspecific patterns of $A b d-B$ gene activity during the blastoderm-early gastrula stage [92-95]. At this point in development one of the key functions of insulators is to prevent cross talk between the adjacent parasegmentspecific initiation elements that can inappropriately activate or silence the cis-regulatory domains flanking the insulator $[67,69,77,96]$. For mutations that disrupt specific insulators, this mix of ectopic activation and silencing can be visualized in the embryo by changes in the level of $A b d-B$ expression [67, 77, 96]. For example, in Fab-6 mutants, the inappropriate activation of the iab-6 cisregulatory domain in PS10 would noticeably upregulate $A b d-B$ expression in this parasegment compared to that in wild type where $i a b-5$ normally directs $A b d-B$ expression [69]. However, the level of $A b d-B$ expression in PS10 in the Fab-6 mutant is not equivalent to that in the adjacent parasegment PS11 because in some of the PS10 cells the iab-5 cis-regulatory domain is inappropriately silenced. In fact, this is the phenotype that we observed in $d C T C F^{m-z-}$ mutant embryos. $A b d-B$ expression is each posterior parasegment was upregulated compared to the corresponding parasegment in wild type, but the extent of upregulation was not equivalent to that in the adjacent more posterior parasegments.

While the opposing phenotypes associated with the loss $d C T C F$ activity at different stages of development argue in favor of the idea that dCTCF is critical to the functioning of the insulators associated with the $A b d-B$ cis-regulatory domains, there was one rather puzzling observation-namely, the apparent effects of the $d C T C F$ GE24185 mutation on the activity of the Fab-7 insulator. In adult GE24185 flies, the partial transformation of A6 (PS11) into A5 (PS10) would point either to a loss of $A b d-B$ promoter activity or the spreading of silencing from iab-7 PRE into iab-6, shutting off this cis-regulatory domain in PS10 (or a combination of both). In the embryo, the upregulation of $A b d-B$ expression in PS11 would require either an increase in $A b d-B$ promoter activity or the ectopic activation of $i a b-7$ by the $i a b-6$ initiator in PS11 cells. While these stage-specific phenotypic effects are more readily explained by a disruption in Fab-7 insulator activity rather than diametrically opposite effects on the $A b d-B$ promoter as development proceeds, $F a b-7$ differs from the other $A b d-B$ insulators in that it does not have $\mathrm{dCTCF}$ binding sites [78]. In this case, the apparent loss of Fab-7 insulator activity would have to be an indirect consequence of disruptions in the functioning of the neighboring dCTCF-dependent insulators, Fab-6 and Fab-8. Although transgene assays have argued that the activity of an insulator depends upon its neighbors (for review see [1]), this would be one of the first examples in an endogenous setting. Further studies will clearly be required to explain the apparent effects on Fab-7 activity.

Additionally, we found that the expression of the Notch pathway gene, insv, was also changed in $d C T C F^{m-z-}$ embryos, while en expression seemed to be 
unaffected. Unlike $A b d-B$, where there are good reasons to believe that the effects on gene regulation are a direct consequence of the loss of $d C T C F$ activity, we do not know whether the effects on insv are direct or indirect. However, it is worth noting that the insv gene and its partner, elba2, are flanked by sequences that are bound by dCTCF, while the potential insulators for the en locus appear to be occupied by $\mathrm{Su}(\mathrm{Hw})$ rather than dCTCF $[56,57]$.

\section{Conclusions}

CTCF is one of the few DNA binding insulator proteins that is conserved in bilaterians. To learn more about its role in chromosome architecture we have identified and characterized the two protein-protein interaction modules in Drosophila dCTCF. The first is responsible for the multimerization of the $\mathrm{dCTCF}$ protein while the second is responsible for interactions between dCTCF and CP190. We have also tested the functioning of proteins lacking these interaction modules in vivo. We found that a dCTCF protein lacking sequences critical for CP190 interactions was almost as effective as wild type in rescuing the phenotypic effects of a $d C T C F$ null allele. In contrast, a dCTCF protein lacking the multimerization domain had only partial functionality and did not fully rescue phenotypic effects of the null allele.

\section{Methods}

\section{Plasmid construction}

For protein purification purposes, protein fragments were either PCR-amplified using corresponding primers (see Additional file 9: Table S2), or digested from dCTCF cDNA (1-288, BamHI-EcoRI; 1-205, BamHI-XhoI; 1163, BamHI-PvuII; 1-125, BamHI-RsaI; 70-163, MboIPvuII; 125-180, RsaI-RsaI) and subcloned into pGEX4T1 (GE Healthcare, Little Chalfont, Buckinghamshire, United Kingdom) or pET32a $(+)$ vector (Merck Biosciences, Darmstadt, Germany) in-frame with corresponding tag. In the case of $6 x$ His-fusions without thioredoxin, its coding sequence was excised from pET32a (+) vector with $N d e I$. A modified pET32a $(+)$ vector was used to express proteins with a TEV-cleavable thioredoxin-6xHis-tag.

For protein expression in S2 cells and for generation of transgenic flies, protein coding sequences were cloned in-frame with 3xFLAG, excised, and subcloned into the Casper vector with Hsp83 promoter [64].

\section{Generation and analysis of transgenic lines}

The construct and P25.7wc plasmid were injected into yacw ${ }^{1118}$ pre-blastoderm embryos [97]. The resultant flies were crossed with $y a c w^{1118}$ flies, and the transgenic progeny were identified by their eye color.
Protein expression and purification, size-exclusion chromatography, and chemical cross-linking

Protein expression and purification were performed using standard procedures. Briefly, BL21 cells were disrupted by sonication in buffer A (40 mM HEPES-KOH pH 7.7, $400 \mathrm{mM} \mathrm{NaCl}, 5 \mathrm{mM} \beta$-mercaptoethanol, $20 \mathrm{mM}$ imidazole) containing $1 \mathrm{mM}$ PMSF and Calbiochem Complete Protease Inhibitor Cocktail VII $(1 \mu \mathrm{L} / 1 \mathrm{ml})$. After centrifugation, lysate was applied to an Ni-NTA column, and, after washing, was eluted with $300 \mathrm{mM}$ imidazole and dialyzed against an appropriate buffer. For cleavage of the thioredoxin-6xHis-tagged protein, TEV protease was added at a molar ratio of 1:50 directly to the eluted protein. The mixture was incubated for $2 \mathrm{~h}$ at room temperature, dialyzed against buffer A, and applied to the Ni-NTA column. Flow-through was collected; dialyzed against 20 mM Tris$\mathrm{HCl} \mathrm{pH} \mathrm{7.4,} \mathrm{and} 1 \mathrm{mM}$ DTT; and further purified using a SOURCE15Q 4.6/100 column (GE Healthcare). Sizeexclusion chromatography was performed as described [61] using Sephacryl S200 16/60 or Superdex 200 10/ 300GL columns (GE Healthcare) in $20 \mathrm{mM}$ Tris- $\mathrm{HCl}$ $\mathrm{pH} 7.4,150 \mathrm{mM} \mathrm{NaCl}$, and $1 \mathrm{mM}$ DTT. The protein concentration was adjusted to $5 \mu \mathrm{M}$ and chemical crosslinking was carried out for $10 \mathrm{~min}$ at room temperature in buffer B containing 20 mM HEPES-KOH pH 7.7, $150 \mathrm{mM}$ $\mathrm{NaCl}, 20 \mathrm{mM}$ imidazole, and $1 \mathrm{mM} \beta$-mercaptoethanol. Cross-linking was quenched with $50 \mathrm{mM}$ glycine and the cross-linked samples were resolved using SDS-PAGE followed by silver-staining. For analysis of complex formation between CP190-BTB and CTCF-CTD, the proteins were purified, dialyzed against buffer $B$, and mixed in corresponding molar ratio, with a constant CP190-BTB concentration of $20 \mu \mathrm{M}$. After incubation at room temperature for $2 \mathrm{~h}$ the protein mixture was cross-linked with glutaraldehyde as described above. Samples were resolved using SDS-PAGE followed by Coomassie staining.

\section{Pull-down assays}

GST-pull-downs were performed with Immobilized Glutathione Agarose (Pierce) in buffer C (20 mM HEPES-KOH pH 7.7, $150 \mathrm{mM} \mathrm{NaCl}, 10 \mathrm{mM} \mathrm{MgCl}_{2}$, $0.1 \mathrm{mM} \mathrm{ZnCl} 2,0.1 \% \mathrm{NP} 40,10 \%$ (w/w) glycerol). BL21 cells were grown in lysogeny broth media to an A600 of 1.0 at $37{ }^{\circ} \mathrm{C}$ and then induced with $1 \mathrm{mM}$ IPTG at $18{ }^{\circ} \mathrm{C}$ overnight. $\mathrm{ZnCl}_{2}$ was added to a final concentration of $100 \mu \mathrm{M}$ before induction. Cells were disrupted by sonication, centrifuged, and applied to resin for $10 \mathrm{~min}$ at room temperature. After binding, the resin was washed two times with buffer $C$. The resin with immobilized protein was then mixed with a solution of interacting protein or Schneider 2 cells nuclear lysate equilibrated in buffer $\mathrm{C}$. Incubation was continued for $2 \mathrm{~h}$ at room temperature (for recombinant proteins) or at $+4{ }^{\circ} \mathrm{C}$ (Schneider 2 cells nuclear lysate). The resin was then 
washed four times with buffer $\mathrm{C}$ containing $500 \mathrm{mM}$ $\mathrm{NaCl}$ and elution performed with $50 \mathrm{mM}$ reduced gluta-

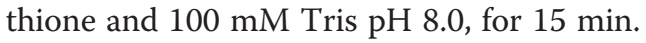

\section{Limited proteolysis}

Thioredoxin-fused CTCF[1-205] protein was purified and dialyzed against $20 \mathrm{mM}$ HEPES- $\mathrm{KOH} \mathrm{pH}$ 7.7, $150 \mathrm{mM} \mathrm{NaCl}, 10 \mathrm{mM} \mathrm{MgCl}$, and $2.5 \mathrm{mM} \mathrm{CaCl}_{2}$. The protein concentration was adjusted to $10 \mu \mathrm{M}$ and indicated amounts of proteinase $\mathrm{K}$ (Fermentas) or trypsin (Sigma) diluted in the same buffer were added. After 10 min incubation at room temperature, PMSF was added to final concentration of $5 \mathrm{mM}$ and incubation continued for a further $10 \mathrm{~min}$. Samples were resolved using SDS-PAGE followed by Coomassie staining. The protein bands were excised and subjected to complete trypsin digestion and MALDI-TOF mass spectrometry. Protein identification and peptide mapping were performed using the MASCOT server (Matrix Science).

\section{Drosophila cells nuclear lysate preparation}

Schneider 2 cells were grown in SFX media, collected by centrifugation at $700 \mathrm{~g}$ for $5 \mathrm{~min}$, washed once with $1 \mathrm{xPBS}$, resuspended in buffer IP-0 $(10 \mathrm{mM}$ Tris- $\mathrm{HCl}$ $\mathrm{pH}$ 8.0, $10 \mathrm{mM} \mathrm{NaCl}, 10 \mathrm{mM} \mathrm{MgCl}_{2}, 1 \mathrm{mM}$ EDTA, 1 mM EGTA, $1 \mathrm{mM}$ DTT, $250 \mathrm{mM}$ sucrose, $1 \mathrm{mM}$ PMSF, $0.2 \%$ NP-40, Calbiochem Protease Inhibitor Cocktail V), incubated for 10 minutes at $+4{ }^{\circ} \mathrm{C}$, and disrupted by 20 strokes in Dounce homogenizer on ice. Nuclei were collected at $3000 \mathrm{~g}$ for $10 \mathrm{~min}$ and resuspended in IP-10+ (10 mM Tris- $\mathrm{HCl} \mathrm{pH}$ 8.0, $10 \mathrm{mM} \mathrm{NaCl}, 10 \mathrm{mM} \mathrm{MgCl}{ }_{2}$, $1 \mathrm{mM}$ EDTA, 1 mM EGTA, $1 \mathrm{mM}$ DTT, 10 \% glycerol, $0.1 \% \mathrm{NP}-40$; in the case of subsequent immunoprecipitation assays, DTT was not used). An equal volume of IP-850+ (10 mM Tris-HCl pH 8.0, $850 \mathrm{mM} \mathrm{NaCl}, 10$ $\mathrm{mM} \mathrm{MgCl}_{2}, 1 \mathrm{mM}$ EDTA, $1 \mathrm{mM}$ EGTA, $1 \mathrm{mM}$ DTT, $10 \%$ glycerol, $0.1 \%$ NP-40) was added and nuclei were lysed for 10 min on ice, after that two volumes of IP-10+ and $1 \mathrm{U} / \mathrm{ml}$ DNAse I were added and incubation was continued for $10 \mathrm{~min}$ in a rotator at room temperature. After centrifugation at $16,000 \mathrm{~g}$ for $20 \mathrm{~min}$, lysate was used in immunoprecipitation or GST-pull-down assays.

\section{Co-immunoprecipitation}

Antibodies were immobilized for $2 \mathrm{~h}$ on pre-equilibrated Protein A or Protein G beads in buffer D (20 mM Tris $\mathrm{pH}$ 7.4, $150 \mathrm{mM} \mathrm{NaCl}, 10 \mathrm{mM} \mathrm{MgCl} 2,0.1 \mathrm{mM} \mathrm{ZnCl}_{2}$, $0.1 \% \mathrm{NP} 40,10 \%(\mathrm{w} / \mathrm{w})$ glycerol). The beads were then washed for $1 \mathrm{~h}$ with the same buffer containing $10 \mathrm{mg} / \mathrm{ml}$ $\mathrm{BSA}$, and incubated overnight at $+4{ }^{\circ} \mathrm{C}$ with nuclear lysate. After incubation with the lysate, the beads were washed four times with buffer D containing $500 \mathrm{mM} \mathrm{NaCl}$ and boiled in SDS-PAGE sample buffer.

\section{Fly extract preparation}

Twenty adult flies were homogenized with a pestle in $200 \mu \mathrm{l}$ of $1 x \mathrm{xBS}$ containing $1 \% \beta$-mercaptoethanol, $10 \mathrm{mM}$ PMSF, and 1:100 Calbiochem Complete Protease Inhibitor Cocktail VII. The suspension was sonicated three times for $5 \mathrm{~s}$ at $5 \mathrm{~W}$. Then, $200 \mu \mathrm{l}$ of $4 \mathrm{xSDS}$-PAGE sample buffer was added and the mixture incubated for $10 \mathrm{~min}$ at $100{ }^{\circ} \mathrm{C}$ and centrifuged at $16,000 \mathrm{~g}$ for $10 \mathrm{~min}$.

\section{Antibodies}

Antibodies were raised against dCTCF[1-163], dCTCF [612-818], and CP190[308-1096] fragments in rabbits and rats. GAGA-factor antibodies were raised against full-length protein in rats. Antibodies were purified from serum by ammonium sulfate fractionation followed by affinity purification using $\mathrm{CNBr}$-activated Sepharose (GE Healthcare) with standard protocols. Other antibodies were anti-FLAG (M2, Sigma), anti-6xHis (GE Healthcare), anti-Abd-B (Iowa Developmental Studies Hybridoma Bank), and anti-Insv (gift of Tsutomu Aoki).

\section{Cell culture, transfection, and dual luciferase assay}

Drosophila S2 cells were grown in SFX medium (HyClone) at $25{ }^{\circ} \mathrm{C}$. Transfection of plasmids was performed with the Cellfectin II reagent (Invitrogen) according to the manufacturer's instructions. Typically, cells were transfected in six-well plates and grown for 24-48 h before harvesting. All transfection procedures were performed with three independent replicates.

The dual luciferase assay was performed with the Firefly \& Renilla Luciferase Assay Kit (Biotium).

\section{Chromatin immunoprecipitation}

Chromatin was prepared from S2 cells and mid-late pupae.

\section{S2 cells}

Formaldehyde from $10 \%$ stock was added to a $1 \%$ final concentration to $10^{7}$ cells in $10 \mathrm{ml}$ of SFX medium and samples were incubated in a rotator at room temperature for $15 \mathrm{~min}$. The cross-linking was stopped by $0.125 \mathrm{M}$ glycine. The samples were placed on ice and washed three times with PBS with $0.5 \mathrm{mM}$ PMSF and centrifuged at $1000 \mathrm{rpm}$ for $5 \mathrm{~min}$ at $4{ }^{\circ} \mathrm{C}$. The pellet was resuspended in $10 \mathrm{ml}$ of buffer I ( 25 mM HEPES pH 7.8, $1.5 \mathrm{mM} \mathrm{MgCl} 2,10 \mathrm{mM} \mathrm{KCl}, 0.1 \% \mathrm{NP} 40,1 \mathrm{mM}$ DTT, 0.5 mM PMSF, Calbiochem Complete (EDTA)-free Protease Inhibitor Cocktail V) and placed on ice for $10 \mathrm{~min}$. The suspension was homogenized in a Dounce homogenizer with pestle " $\mathrm{B}$ " 20 times and centrifuged at $2000 \mathrm{rpm}$ for $5 \mathrm{~min}$ at $4{ }^{\circ} \mathrm{C}$. The pellet was resuspended in $3 \mathrm{ml}$ of buffer II (50 mM HEPES pH 7.8, $140 \mathrm{mM}$ $\mathrm{NaCl}, 1 \mathrm{mM}$ EDTA, 1 \% Triton X-100, 0.1 \% sodium deoxycholate, $0.1 \%$ SDS, 0.5 mM PMSF, Calbiochem Complete (EDTA)-free Protease Inhibitor Cocktail V) 
and sonicated in a Bioruptor sonifier (40 alternating 30-s $\mathrm{ON}$ and 60 -s OFF intervals). Finally, $50-\mu \mathrm{L}$ aliquots were used to test the extent of sonication and to measure DNA concentration.

\section{Pupae}

A 500-mg pupa sample was ground in a mortar in liquid nitrogen and resuspended in $10 \mathrm{~mL}$ of buffer $\mathrm{A}(15 \mathrm{mM}$ HEPES-KOH pH 7.6, $60 \mathrm{mM} \mathrm{KCl,} 15 \mathrm{mM} \mathrm{NaCl}, 13 \mathrm{mM}$ EDTA, $0.1 \mathrm{mM}$ EGTA, $0.15 \mathrm{mM}$ spermine, $0.5 \mathrm{mM}$ spermidine, $0.5 \%$ NP-40, $0.5 \mathrm{mM}$ DTT) supplemented with $0.5 \mathrm{mM}$ PMSF and Calbiochem Protease Inhibitor Cocktail V. The suspension was then homogenized in a Dounce homogenizer with pestle "B" and filtered through Nylon Cell Strainer (BD Biosciences, USA). The homogenate was transferred to $3 \mathrm{~mL}$ of buffer A with $10 \%$ sucrose (AS), and the nuclei were pelleted by centrifugation at $4000 \mathrm{~g}$ for $5 \mathrm{~min}$ at $4{ }^{\circ} \mathrm{C}$. The pellet was resuspended in $5 \mathrm{~mL}$ of buffer A, homogenized again in a Dounce homogenizer, and transferred to $1.5 \mathrm{~mL}$ of buffer AS to collect the nuclei by centrifugation. The nuclear pellet was resuspended in wash buffer (15 mM HEPES-KOH pH 7.6, $60 \mathrm{mM} \mathrm{KCl}, 15 \mathrm{mM} \mathrm{NaCl}, 1 \mathrm{mM}$ EDTA, $0.1 \mathrm{mM}$ EGTA, $0.1 \%$ NP-40, protease inhibitors) and cross-linked with $1 \%$ formaldehyde for $15 \mathrm{~min}$ at room temperature. Crosslinking was stopped by adding glycine to a final concentration of $125 \mathrm{mM}$. The nuclei were washed with three 10$\mathrm{mL}$ portions of wash buffer and resuspended in $1.5 \mathrm{~mL}$ of nuclear lysis buffer (15 mM HEPES pH 7.6, $140 \mathrm{mM} \mathrm{NaCl}$, 1 mM EDTA, 0.1 mM EGTA, 1 \% Triton X-100, 0.5 mM DTT, $0.1 \%$ sodium deoxycholate, $0.1 \%$ SDS, protease inhibitors). The suspension was sonicated in a Bioruptor sonifier (35 alternating 30-s ON and 60-s OFF intervals), and $50-\mu \mathrm{L}$ aliquots were used to test the extent of sonication and to measure DNA concentration.

\section{Immunoprecipitation}

Debris was removed by centrifugation at $14,000 \mathrm{~g}$ for $10 \mathrm{~min}$ at $4{ }^{\circ} \mathrm{C}$, and chromatin was pre-cleared with Protein A agarose (Pierce) blocked with BSA and salmon sperm DNA, with $50-\mu \mathrm{L}$ aliquots of such pre-cleared chromatin being stored as input material. Samples containing $10-20 \mu \mathrm{g}$ of DNA equivalent in $1 \mathrm{~mL}$ of nuclear lysis buffer were incubated overnight at $4{ }^{\circ} \mathrm{C}$ with rabbit antibodies against dCTCF (1:500) and CP190 (1:1000), mouse antibodies against FLAGx3 (1:200), or with non-specific IgG purified from rabbit or mouse pre-immune sera (control). Chromatin-antibody complexes were collected using blocked Protein A or G agarose at $4{ }^{\circ} \mathrm{C}$ over $5 \mathrm{~h}$. After several rounds of washing with lysis buffer (as such and with $500 \mathrm{mM} \mathrm{NaCl}$ ), LiCl buffer (20 mM Tris- $\mathrm{HCl} \mathrm{pH} 8$, $250 \mathrm{mM}$ LiCl, 1 mM EDTA, 0.5 \% NP-40, $0.5 \%$ sodium deoxycholate, protease inhibitors), and TE buffer (10 mM Tris- $\mathrm{HCl} \mathrm{pH} 8,1 \mathrm{mM}$ EDTA), the DNA was eluted with elution buffer (50 mM Tris- $\mathrm{HCl}, \mathrm{pH}$ 8.0, 1 mM EDTA, $1 \%$ SDS), the cross-links were reversed, and the precipitated DNA was extracted by the phenol-chloroform method. The enrichment of specific DNA fragments was analyzed by real-time PCR, using a StepOne Plus Thermal Cycler (Applied Biosystems). The primers used for PCR in ChIP experiments for genome fragments are shown in Additional file 9: Table S2.

\section{Additional files}

Additional file 1: Figure S1. (A) Multiple sequence alignment of CTCF homologs in distant species. (B) Multiple sequence alignment of dCTCF proteins from different Drosophila species. (TIFF $15876 \mathrm{~kb}$ )

Additional file 2: Figure S2. (A) Glutaraldehyde cross-linking of different dCTCF N-terminal derivatives as indicated (see also schematic in Fig. 1). Also included in panel A is glutaraldehyde cross-linking of the dCTCF-CTD sequence 612-818. (B) Limited proteolysis of the thioredoxin-fused dCTCF[1-205] protein with proteinase K or trypsin. Proteolysis-resistant fragments (indicated by the frame) were excised from the gel and subjected to MALDI-TOF mass spectrometry. Peptides found in these bands are indicated in the dCTCF N-terminal amino-acid sequence. Peptides recovered in the proteinase $\mathrm{K}$ digestion are underlined while peptides from trypsin digestion are shown in bold. (TIFF $4642 \mathrm{~kb}$ )

Additional file 3: Table S1. The results of limited proteinase $K$ or trypsin digestion. (DOC $36 \mathrm{~kb}$ )

Additional file 4: Figure S3. Diagram showing the dCTCF gene and site of insertion of the GE24185 transposon. Also included is the sequence of the wild-type dCTCF gene flanking the transposon insertion site and the sequences of the two excision derivatives GEx52 and GEx56. (TIFF 2489 kb)

Additional file 5: Figure S4. Abdominal transformations in females. Posterior sternites of wild type, GE24185, and GE24185 females rescued with the hsp83:dCTCF ${ }^{+}$, hsp83:dCTCF ${ }^{\Delta N}$, or hsp83:dCTCF ${ }^{\Delta C}$ transgenes. Upper panel shows dark field images of the sternites $A 6$ and A7, and lower panel shows the same sternites in bright field. The bristles on the A7 sternite of GE24185 differ from wild type in that they are arranged in a manner that is characteristic of A6 (pointing outward rather than inward), and the trichome pattern of A7, evident in the dark field, likewise is characteristic of A6. This phenotypic transformation is rescued by the hsp83:dCTCF ${ }^{+}$and ssp83:dCTCF $^{\Delta C}$ transgenes, but not by the hsp83:dCTCF ${ }^{\Delta N}$ transgene. (TIFF $3997 \mathrm{~kb}$ )

Additional file 6: Figure S5. Histograms showing dCTCF occupancy in chromatin isolated from mid-late pupa at sequences corresponding to the BX-C insulators Fab-3, Fab-4, Mcp, Fab-6, Fab-8, the Abd-D promoter, and several other dCTCF insulators (9A1, 21E2, 24C4, 27B2, and 57B4R). Chromatin was prepared from $2-3$ day old wild type $\left(W T ; y^{\top} W^{1118}\right)$ and homozyogous GE24184 (GE/GE) mutant pupae. After fixation and processing, the isolated chromatin was incubated with antibodies directed against the C-terminal region of dCTCF. Sequences from tub, rpl32, and 62D regions were used as negative controls for dCTCF binding. The axis shows the scale for dCTCF enrichment. Error bars show standard deviations of quadruplicate PCR measurements in two biological replicates. The results are presented as a percentage of input DNA. (TIFF $2160 \mathrm{~kb}$ )

Additional file 7: Figure S6. Western blots of protein extracts prepared from wild-type ( $w t ; y^{7} w^{1118}$ ) and homozygous GE24185 mutant pupae. The blots were probed with CP190, N-dCTCF, Pita antibodies. Protein levels in each extract were visualized by staining the membrane after protein transfer with Ponceau S. (TIFF $4911 \mathrm{~kb}$ )

Additional file 8: Figure S7. Western blots of protein extracts from wild-type flies $\left(w t ; y^{7} w^{7}\right)$ and homozygous GE24185 flies carrying the hsp83:dCTCF $F^{+}$, hsp83:dCTCF ${ }^{\Delta N}$, or hsp83:dCTCF ${ }^{\Delta C}$ dCTCF transgenes. All three of the transgene encoded proteins have an N-terminal 3xFLAG-tag and are detected with FLAG antibodies. The respective transgenes are indicated in the figure. (TIFF $668 \mathrm{~kb}$ )

Additional file 9: Table S2. List of primers used. (DOC 51 kb) 


\section{Competing interests}

The authors declare that they have no competing interests.

\section{Authors' contributions}

$A B$ performed protein purification and analysis. OM carried out ChIP and cell culture assays. OK performed molecular and genetic fly analysis. TI did yeast two-hybrid analysis. VM made Drosophila cuticle preparations. GD and DW performed antibody staining and analysis. PS and PG conceived the study, helped with the experimental design and wrote the manuscript. All authors participated in drafting the manuscript. All authors read and approved the final manuscript.

\section{Acknowledgements}

The authors would like to thank Tsutomu Aoki for the gift of Insv polyclonal antibodies. This study was supported by the Ministry of Education and Science of the Russian Federation (project no. 14.B25.31.0022) and a grant from NIH GM043432 to PS, and Russian Foundation for Basic Research (projects no. 13-04-00340 and 13-04-40136-n) and RF Presidential Stipend no. SP- 4081.2015.4 to OM.

\section{Author details}

'Department of the Control of Genetic Processes, Institute of Gene Biology, Russian Academy of Sciences, Moscow, Russia. ' Laboratory of Gene Expression Regulation in Development, Institute of Gene Biology, Russian Academy of Sciences, Moscow, Russia. ${ }^{3}$ Department of Molecular Biology, Princeton University, Princeton, NJ, USA. ${ }^{4}$ Department of Biology, Nikolaev V.A. Sukhomlinsky National University, Nikolaev, Ukraine.

\section{Received: 15 May 2015 Accepted: 15 July 2015}

\section{Published online: 07 August 2015}

\section{References}

1. Chetverina D, Aoki T, Erokhin M, Georgiev P, Schedl P. Making connections: insulators organize eukaryotic chromosomes into independent cis-regulatory networks. Bioessays. 2014;36:163-72.

2. Tanay A, Cavalli G. Chromosomal domains: epigenetic contexts and functional implications of genomic compartmentalization. Curr Opin Genet Dev. 2013;23:197-203.

3. Krijger PH, de Laat W. Identical cells with different 3D genomes; cause and consequences? Curr Opin Genet Dev. 2013;23:191-6.

4. Wendt KS, Grosveld FG. Transcription in the context of the 3D nucleus. Curr Opin Genet Dev. 2014;25:62-7.

5. Nora EP, Dekker J, Heard E. Segmental folding of chromosomes: a basis for structural and regulatory chromosomal neighborhoods? Bioessays. 2013;35:818-28.

6. Gibcus JH, Dekker J. The hierarchy of the 3D genome. Mol Cell. 2013;49:773-82.

7. Kuhn EJ, Geyer PK. Genomic insulators: connecting properties to mechanism. Curr Opin Cell Biol. 2003;15:259-65.

8. Brasset $E$, Vaury C. Insulators are fundamental components of the eukaryotic genomes. Heredity. 2005;94:571-6.

9. Raab JR, Kamakaka RT. Insulators and promoters: closer than we think. Nat Rev Genet. 2010;11:439-46.

10. Matzat LH, Lei EP. Surviving an identity crisis: a revised view of chromatin insulators in the genomics era. Biochim Biophys Acta. 1839;2014:203-14.

11. Schoborg T, Labrador M. Expanding the roles of chromatin insulators in nuclear architecture, chromatin organization and genome function. Cell Mol Life Sci. 2014;71:4089-113.

12. Kyrchanova O, Georgiev P. Chromatin insulators and long-distance interactions in Drosophila. FEBS Lett. 2014;588:8-14.

13. Maksimenko O, Georgiev P. Mechanisms and proteins involved in long-distance interactions. Front Genet. 2014;5:28.

14. Cleard F, Moshkin Y, Karch F, Maeda RK. Probing long-distance regulatory interactions in the Drosophila melanogaster bithorax complex using Dam identification. Nat Genet. 2006;38:931-5.

15. Kyrchanova O, Chetverina D, Maksimenko O, Kullyev A, Georgiev P. Orientation-dependent interaction between Drosophila insulators is a property of this class of regulatory elements. Nucleic Acids Res. 2008;36:7019-28.

16. Kyrchanova O, Ivlieva T, Toshchakov S, Parshikov A, Maksimenko O, Georgiev P. Selective interactions of boundaries with upstream region of Abd-B promoter in Drosophila bithorax complex and role of dCTCF in this process. Nucleic Acids Res. 2011;39:3042-52.

17. Fujioka M, Sun G, Jaynes JB. The Drosophila eve insulator Homie promotes eve expression and protects the adjacent gene from repression by polycomb spreading. PLoS Genet. 2013;9:e1003883.

18. Junier I, Dale RK, Hou C, Kepes F, Dean A. CTCF-mediated transcriptional regulation through cell type-specific chromosome organization in the beta-globin locus. Nucleic Acids Res. 2012;40:7718-27.

19. Kim A, Dean A. Chromatin loop formation in the beta-globin locus and its role in globin gene transcription. Mol Cells. 2012;34:1-5.

20. Erokhin M, Davydova A, Kyrchanova O, Parshikov A, Georgiev P, Chetverina D. Insulators form gene loops by interacting with promoters in Drosophila. Development. 2011;138:4097-106.

21. Kyrchanova O, Maksimenko O, Stakhov V, Ivlieva T, Parshikov A, Studitsky VM, et al Effective blocking of the white enhancer requires cooperation between two main mechanisms suggested for the insulator function. PLoS Genet. 2013;9:e1003606.

22. Bell $A C$, West $A G$, Felsenfeld $G$. The protein CTCF is required for the enhancer blocking activity of vertebrate insulators. Cell. 1999;98:387-96.

23. Gray CE, Coates CJ. Cloning and characterization of CDNAs encoding putative CTCFs in the mosquitoes, Aedes aegypti and Anopheles gambiae. BMC Mol Biol. 2005;6:16.

24. Heger P, Marin B, Schierenberg E. Loss of the insulator protein CTCF during nematode evolution. BMC Mol Biol. 2009;10:84

25. Heger P, Marin B, Bartkuhn M, Schierenberg E, Wiehe T. The chromatin insulator CTCF and the emergence of metazoan diversity. Proc Natl Acad Sci U S A. 2012;109:17507-12.

26. Filippova GN, Fagerlie S, Klenova EM, Myers C, Dehner Y, Goodwin G, et al. An exceptionally conserved transcriptional repressor, CTCF, employs different combinations of zinc fingers to bind diverged promoter sequences of avian and mammalian c-myc oncogenes. Mol Cell Biol. 1996;16:2802-13.

27. Klenova EM, Nicolas RH, Paterson HF, Carne AF, Heath CM, Goodwin GH, et al. CTCF, a conserved nuclear factor required for optimal transcriptional activity of the chicken c-myc gene, is an 11-Zn-finger protein differentially expressed in multiple forms. Mol Cell Biol. 1993;13:7612-24.

28. Merkenschlager M, Odom DT. CTCF and cohesin: linking gene regulatory elements with their targets. Cell. 2013;152:1285-97.

29. Ohlsson R, Lobanenkov V, Klenova E. Does CTCF mediate between nuclear organization and gene expression? Bioessays. 2010;32:37-50.

30. Ghirlando R, Giles K, Gowher H, Xiao T, Xu Z, Yao H, et al. Chromatin domains, insulators, and the regulation of gene expression. Biochim Biophys Acta. 1819;2012:644-51.

31. Zuin J, Dixon JR, van der Reijden MI, Ye Z, Kolovos P, Brouwer RW, et al. Cohesin and CTCF differentially affect chromatin architecture and gene expression in human cells. Proc Natl Acad Sci U S A. 2014;111:996-1001.

32. Handoko L, Xu H, Li G, Ngan CY, Chew E, Schnapp M, et al. CTCF-mediated functional chromatin interactome in pluripotent cells. Nat Genet. 2011;43:630-8.

33. Dixon JR, Selvaraj S, Yue F, Kim A, Li Y, Shen Y, et al. Topological domains in mammalian genomes identified by analysis of chromatin interactions. Nature. 2012;485:376-80.

34. Sanyal A, Lajoie BR, Jain G, Dekker J. The long-range interaction landscape of gene promoters. Nature. 2012;489:109-13.

35. Kyrchanova O, Toshchakov S, Podstreshnaya Y, Parshikov A, Georgiev P. Functional interaction between the Fab-7 and Fab-8 boundaries and the upstream promoter region in the Drosophila Abd-B gene. Mol Cell Biol. 2008;28:4188-95.

36. Herold M, Bartkuhn M, Renkawitz R. CTCF: insights into insulator function during development. Development. 2012;139:1045-57.

37. Moon H, Filippova G, Loukinov D, Pugacheva E, Chen Q, Smith ST, et al. CTCF is conserved from Drosophila to humans and confers enhancer blocking of the Fab-8 insulator. EMBO Rep. 2005;6:165-70.

38. Wendt KS, Yoshida K, Itoh T, Bando M, Koch B, Schirghuber E, et al. Cohesin mediates transcriptional insulation by CCCTC-binding factor. Nature. 2008;451:796-801.

39. Parelho V, Hadjur S, Spivakov M, Leleu M, Sauer S, Gregson HC, et al. Cohesins functionally associate with CTCF on mammalian chromosome arms. Cell. 2008;132:422-33.

40. Rubio ED, Reiss DJ, Welcsh PL, Disteche CM, Filippova GN, Baliga NS, et al. CTCF physically links cohesin to chromatin. Proc Natl Acad Sci U S A. 2008;105:8309-14.

41. Stedman W, Kang H, Lin S, Kissil JL, Bartolomei MS, Lieberman PM. Cohesins localize with CTCF at the KSHV latency control region and at cellular c-myc and H19/lgf2 insulators. Embo J. 2008;27:654-66. 
42. Heidari N, Phanstiel DH, He C, Grubert F, Jahanbani F, Kasowski M, et al. Genome-wide map of regulatory interactions in the human genome. Genome Res. 2014;24:1905-17.

43. Jin F, Li Y, Dixon JR, Selvaraj S, Ye Z, Lee AY, et al. A high-resolution map of the three-dimensional chromatin interactome in human cells. Nature. 2013;503:290-4.

44. Dorsett D. Cohesin: genomic insights into controlling gene transcription and development. Curr Opin Genet Dev. 2011;21:199-206.

45. Xiao T, Wallace J, Felsenfeld G. Specific sites in the C terminus of CTCF interact with the SA2 subunit of the cohesin complex and are required for cohesin-dependent insulation activity. Mol Cell Biol. 2011;31:2174-83.

46. Misulovin Z, Schwartz YB, Li XY, Kahn TG, Gause M, MacArthur S, et al. Association of cohesin and Nipped-B with transcriptionally active regions of the Drosophila melanogaster genome. Chromosoma. 2008;117:89-102.

47. Ahanger SH, Srinivasan A, Vasanthi D, Shouche YS, Mishra RK. Conserved boundary elements from the Hox complex of mosquito, Anopheles gambiae. Nucleic Acids Res. 2013;41:804-16.

48. Whitfield WG, Chaplin MA, Oegema K, Parry H, Glover DM. The 190 kDa centrosome-associated protein of Drosophila melanogaster contains four zinc finger motifs and binds to specific sites on polytene chromosomes. J Cell Sci. 1995;108:3377-87.

49. Kellogg DR, Field CM, Alberts BM. Identification of microtubule-associated proteins in the centrosome, spindle, and kinetochore of the early Drosophila embryo. J Cell Biol. 1989;109:2977-91.

50. Butcher RD, Chodagam S, Basto R, Wakefield JG, Henderson DS, Raff JW, et al. The Drosophila centrosome-associated protein CP190 is essential for viability but not for cell division. J Cell Sci. 2004;117:1191-9.

51. Vogelmann J, Le Gall A, Dejardin S, Allemand F, Gamot A, Labesse G, et al. Chromatin insulator factors involved in long-range DNA interactions and their role in the folding of the Drosophila genome. PLoS Genet. 2014;10:e1004544.

52. Pai CY, Lei EP, Ghosh D, Corces VG. The centrosomal protein CP190 is a component of the gypsy chromatin insulator. Mol Cell. 2004;16:737-48.

53. Moshkovich N, Nisha P, Boyle PJ, Thompson BA, Dale RK, Lei EP. RNAiindependent role for Argonaute2 in CTCF/CP190 chromatin insulator function. Genes Dev. 2011;25:1686-701.

54. Gerasimova TI, Lei EP, Bushey AM, Corces VG. Coordinated control of dCTCF and gypsy chromatin insulators in Drosophila. Mol Cell. 2007;28:761-72.

55. Mohan M, Bartkuhn M, Herold M, Philippen A, Heinl N, Bardenhagen I, et al. The Drosophila insulator proteins CTCF and CP190 link enhancer blocking to body patterning. EMBO J. 2007;26:4203-14.

56. Schwartz YB, Linder-Basso D, Kharchenko PV, Tolstorukov MY, Kim M, Li HB, et al. Nature and function of insulator protein binding sites in the Drosophila genome. Genome Res. 2012;22:2188-98.

57. Negre N, Brown CD, Shah PK, Kheradpour P, Morrison CA, Henikoff JG, et al. A comprehensive map of insulator elements for the Drosophila genome. PLoS Genet. 2010;6:e1000814

58. Yusufzai TM, Tagami H, Nakatani Y, Felsenfeld G. CTCF tethers an insulator to subnuclear sites, suggesting shared insulator mechanisms across species. Mol Cell. 2004;13:291-8.

59. Pant V, Kurukuti S, Pugacheva E, Shamsuddin S, Mariano P, Renkawitz R, et al. Mutation of a single CTCF target site within the H19 imprinting control region leads to loss of lgf2 imprinting and complex patterns of de novo methylation upon maternal inheritance. Mol Cell Biol. 2004:24:3497-504.

60. Oliver D, Sheehan B, South H, Akbari O, Pai CY. The chromosomal association/dissociation of the chromatin insulator protein Cp190 of Drosophila melanogaster is mediated by the BTB/POZ domain and two acidic regions. BMC Cell Biol. 2010;11:101.

61. Bonchuk A, Denisov S, Georgiev P, Maksimenko O. Drosophila BTB/POZ domains of "ttk group" can form multimers and selectively interact with each other. J Mol Biol. 2011;412:423-36.

62. Ahmad KF, Melnick A, Lax S, Bouchard D, Liu J, Kiang CL, et al. Mechanism of SMRT corepressor recruitment by the BCL6 BTB domain. Mol Cell. 2003;12:1551-64.

63. MacDonald WA, Menon D, Bartlett NJ, Sperry GE, Rasheva V, Meller V, et al. The Drosophila homolog of the mammalian imprint regulator, CTCF, maintains the maternal genomic imprint in Drosophila melanogaster. BMC Biol. 2010;8:105.

64. Greenberg AJ, Schedl P. GAGA factor isoforms have distinct but overlapping functions in vivo. Mol Cell Biol. 2001;21:8565-74.

65. Bhat KM, Farkas G, Karch F, Gyurkovics H, Gausz J, Schedl P. The GAGA factor is required in the early Drosophila embryo not only for transcriptional regulation but also for nuclear division. Development. 1996;122:1113-24.
66. Rorth P. A modular misexpression screen in Drosophila detecting tissue-specific phenotypes. Proc Natl Acad Sci U S A. 1996;93:12418-22.

67. Barges S, Mihaly J, Galloni M, Hagstrom K, Muller M, Shanower G, et al. The Fab-8 boundary defines the distal limit of the bithorax complex iab-7 domain and insulates iab-7 from initiation elements and a PRE in the adjacent iab-8 domain. Development. 2000;127:779-90.

68. Karch F, Galloni M, Sipos L, Gausz J, Gyurkovics H, Schedl P. Mcp and Fab-7: molecular analysis of putative boundaries of cis-regulatory domains in the bithorax complex of Drosophila melanogaster. Nucleic Acids Res. 1994;22:3138-46.

69. lampietro C, Gummalla M, Mutero A, Karch F, Maeda RK. Initiator elements function to determine the activity state of BX-C enhancers. PLoS Genet. 2010;6:e1001260.

70. Perez-Lluch S, Cuartero S, Azorin F, Espinas ML. Characterization of new regulatory elements within the Drosophila bithorax complex. Nucleic Acids Res. 2008;36:6926-33.

71. Smith ST, Wickramasinghe P, Olson A, Loukinov D, Lin L, Deng J, et al. Genome wide ChIP-chip analyses reveal important roles for CTCF in Drosophila genome organization. Dev Biol. 2009;328:518-28.

72. Gruzdeva N, Kyrchanova O, Parshikov A, Kullyev A, Georgiev P. The Mcp element from the bithorax complex contains an insulator that is capable of pairwise interactions and can facilitate enhancer-promoter communication. Mol Cell Biol. 2005;25:3682-9.

73. Hagstrom K, Muller M, Schedl P. Fab-7 functions as a chromatin domain boundary to ensure proper segment specification by the Drosophila bithorax complex. Genes Dev. 1996;10:3202-15.

74. Zhou J, Barolo S, Szymanski P, Levine M. The Fab-7 element of the bithorax complex attenuates enhancer-promoter interactions in the Drosophila embryo. Genes Dev. 1996;10:3195-201.

75. Zhou J, Levine M. A novel cis-regulatory element, the PTS, mediates an anti-insulator activity in the Drosophila embryo. Cell. 1999;99:567-75.

76. Zhou J, Ashe H, Burks C, Levine M. Characterization of the transvection mediating region of the abdominal-B locus in Drosophila. Development. 1999;126:3057-65.

77. Mihaly J, Barges S, Sipos L, Maeda R, Cleard F, Hogga I, et al. Dissecting the regulatory landscape of the $A b d-B$ gene of the bithorax complex. Development. 2006;133:2983-93.

78. Holohan EE, Kwong C, Adryan B, Bartkuhn M, Herold M, Renkawitz R, et al. CTCF genomic binding sites in Drosophila and the organisation of the bithorax complex. PLoS Genet. 2007;3:e112.

79. Maksimenko O, Kyrchanova O, Bonchuk A, Stakhov V, Parshikov A, Georgiev P. Highly conserved ENY2/Sus1 protein binds to Drosophila CTCF and is required for barrier activity. Epigenetics. 2014;9:1261-70.

80. Jeong S, Rokas A, Carroll SB. Regulation of body pigmentation by the Abdominal-B Hox protein and its gain and loss in Drosophila evolution. Cell. 2006;125:1387-99.

81. DiNardo S, Sher E, Heemskerk-Jongens J, Kassis JA, O'Farrell PH. Two-tiered regulation of spatially patterned engrailed gene expression during Drosophila embryogenesis. Nature. 1988;332:604-9.

82. Duan H, Dai Q, Kavaler J, Bejarano F, Medranda G, Negre N, et al. Insensitive is a corepressor for Suppressor of Hairless and regulates Notch signalling during neural development. EMBO J. 2011;30:3120-33.

83. Delorenzi M, Bienz M. Expression of Abdominal-B homeoproteins in Drosophila embryos. Development. 1990;108:323-9.

84. Boulet AM, Lloyd A, Sakonju S. Molecular definition of the morphogenetic and regulatory functions and the cis-regulatory elements of the Drosophila Abd-B homeotic gene. Development. 1991;111:393-405.

85. Holwerda SJ, de Laat W. CTCF: the protein, the binding partners, the binding sites and their chromatin loops. Philos Trans R Soc Lond B Biol Sci. 2013;368:20120369.

86. Martinez SR, Miranda JL. CTCF terminal segments are unstructured. Protein Sci. 2010;19:1110-6.

87. Maksimenko O, Bartkuhn M, Stakhov V, Herold M, Zolotarev N, Jox T, et al. Two new insulator proteins, Pita and ZIPIC, target CP190 to chromatin. Genome Res. 2015;25:89-99.

88. Maeda RK, Karch F. The ABC of the BX-C: the bithorax complex explained. Development. 2006;133:1413-22.

89. Maeda RK, Karch F. Making connections: boundaries and insulators in Drosophila. Curr Opin Genet Dev. 2007;17:394-9.

90. Paro R. Imprinting a determined state into the chromatin of Drosophila. Trends Genet. 1990;6:416-21. 
91. Pirrotta V. Polycomb silencing and the maintenance of stable chromatin states. Results Probl Cell Differ. 1999;25:205-28.

92. Ingham PW, Martinez-Arias A. The correct activation of Antennapedia and bithorax complex genes requires the fushi tarazu gene. Nature. 1986;324:592-7.

93. Muller J, Bienz M. Sharp anterior boundary of homeotic gene expression conferred by the fushi tarazu protein. Embo J. 1992;11:3653-61.

94. Zhang CC, Bienz M. Segmental determination in Drosophila conferred by hunchback (hb), a repressor of the homeotic gene Ultrabithorax (Ubx). Proc Natl Acad Sci U S A. 1992;89:7511-5.

95. Qian S, Capovilla M, Pirrotta V. The bx region enhancer, a distant cis-control element of the Drosophila Ubx gene and its regulation by hunchback and other segmentation genes. Embo J. 1991;10:1415-25.

96. Mihaly J, Hogga I, Gausz J, Gyurkovics H, Karch F. In situ dissection of the Fab-7 region of the bithorax complex into a chromatin domain boundary and a Polycomb-response element. Development. 1997;124:1809-20.

97. Karess RE, Rubin GM. Analysis of $\mathrm{P}$ transposable element functions in Drosophila. Cell. 1984;38:135-46.

\section{Submit your next manuscript to BioMed Central and take full advantage of:}

- Convenient online submission

- Thorough peer review

- No space constraints or color figure charges

- Immediate publication on acceptance

- Inclusion in PubMed, CAS, Scopus and Google Scholar

- Research which is freely available for redistribution 